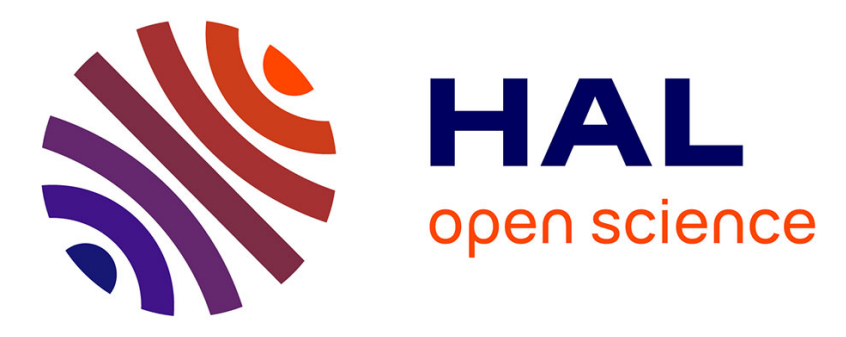

\title{
Time-resolved integrated digital image correlation
}

\author{
J. Neggers, J. P. M. Hoefnagels, M. G. D. Geers, F. Hild, S. Roux
}

\section{To cite this version:}

J. Neggers, J. P. M. Hoefnagels, M. G. D. Geers, F. Hild, S. Roux. Time-resolved integrated digital image correlation. International Journal for Numerical Methods in Engineering, 2015, 103 (3), pp.157182. $10.1002 /$ nme.4882 . hal-01218659

\section{HAL Id: hal-01218659 \\ https://hal.science/hal-01218659}

Submitted on 23 Oct 2015

HAL is a multi-disciplinary open access archive for the deposit and dissemination of scientific research documents, whether they are published or not. The documents may come from teaching and research institutions in France or abroad, or from public or private research centers.
L'archive ouverte pluridisciplinaire HAL, est destinée au dépôt et à la diffusion de documents scientifiques de niveau recherche, publiés ou non, émanant des établissements d'enseignement et de recherche français ou étrangers, des laboratoires publics ou privés. 


\title{
Time-Resolved Integrated Digital Image Correlation
}

\author{
J. Neggers ${ }^{1}$, J.P.M. Hoefnagels ${ }^{1 *}$, M.G.D.Geers ${ }^{1}$, F. Hild ${ }^{2}$ and S. Roux ${ }^{2}$ \\ ${ }^{1}$ Eindhoven University of Technology, Department of Mechanical Engineering, 5612 AZ Eindhoven, \\ Netherlands \\ ${ }^{2}$ LMT Cachan, ENS Cachan / CNRS / PRES UniverSud Paris, 61 avenue du Président Wilson, 94235 \\ Cachan Cedex, France
}

\begin{abstract}
SUMMARY
This paper discusses a method that provides the direct identification of constitutive model parameters by intimately integrating the Finite Element Method (FEM) with Digital Image Correlation (DIC), namely, directly connecting the experimentally obtained images for all time increments to the unknown material parameters. The problem is formulated as a single minimization problem that incorporates all the experimental data. It allows for precise specification of the unknowns, which can be, but are not limited to, the unknown material properties. The tight integration between FEM and DIC enables for identification while providing necessary regularization of the DIC procedure, making the method robust and noise insensitive. Through this approach, the versatility of the FE method is extended to the experimental realm, enhancing the analyses of existing experiments and opening new experimental opportunities.
\end{abstract}

Received ...

KEY WORDS: Digital Image Correlation; Identification; Inverse Methods, Material Characterization

\section{INTRODUCTION}

Accurate prediction of complex mechanical phenomena, such as viscoplasticity, interface delamination, or material failure, enables for the design of advanced materials, systems, and devices with novel mechanical functionality or improved material properties, processed through smart (thermo-)mechanical manufacturing routes. Predictive simulations accounting for complex mechanical behaviors require accurate constitutive models with often many material parameters that need to be determined experimentally. For instance, it is not uncommon that nonlinear material models include more than ten parameters [1-4]. To correctly identify these parameters, traditionally, many individual mechanical experiments would have to be performed, each of which sensitive to certain parameters.

With the advance of real-time in-situ full-field acquisition techniques (e.g. optical photography, electron microscopy, x-ray tomography [5]) it is now increasingly realistic to identify all model parameters from a single mechanical test in which the evolving displacement fields are captured in a sequence of images. However, such a full-field inverse parameter identification strategy remains challenging, because the influence of a parameter on the displacement field is often of the order of the image acquisition noise. Therefore, the algorithm to extract the underlying displacement field from the noisy image sequence should employ

\footnotetext{
*Correspondence to: j.p.m.hoefnages@tue.nl, Eindhoven University of Technology, Department of Mechanical Engineering, 5612 AZ Eindhoven, Netherlands
} 
as few degrees of freedom (dof) as possible to enhance accuracy and robustness. Ideally, by incorporating constraints on the mechanical behavior in the identification algorithm, it becomes possible to reduce the number of dof to the number of material parameters in the constitutive model. Such an optimal one-step parameter identification routine is now feasible, by correlating the image patterns using only those kinematically-admissible 'deformation modes' that correspond to the change of a single model parameter.

In the literature, various full-field identification strategies have been proposed (e.g. [3, 5-12]). However, the majority of the proposed methodologies follow an indirect two-step approach, namely, $(i)$ the experimental displacement field is first measured, and (ii) an inverse procedure is adopted to determine the model parameters by minimizing the difference between the simulated field and its experimental counterpart.

With respect to step $(i)$, Digital Image Correlation (DIC) is usually applied to obtain the displacement field. However, DIC methods are inherently ill-posed because they aim at obtaining a vector field (i.e. the displacement field) from a scalar field (i.e. the gray level pattern). Additionally, the presence of image acquisition noise adds further difficulties to the ill-posedness. The solution to this problem is to reduce the number of unknowns that describe the displacement field. This can be carried out by regularizing the displacement field with a set of interpolation functions, which are chosen to span either local zones of interest (or subimages [13-20]) or the global region of interest [21]. Typically, a wider support of the interpolation functions increases the noise robustness at the price of reducing the displacement resolution. Alternative approaches have been proposed, such as the equilibrium gap method, that decouple the regularization from the applied DIC discretization [22]. Note that all these techniques to determine the displacement field typically do not exploit prior knowledge of the kinematics and, therefore, a relatively large number of interpolation functions (i.e. DOFs) is still needed to describe the displacement field, especially for cases where nonlinear material properties are investigated.

Regarding step (ii), the most straightforward and well-known inverse parameter identification approach is Finite Element Method Updating (FEMU), where the difference between FEM generated displacement fields and measured displacement fields is minimized by iteratively optimizing the unknown material parameters [23]. Many alternative and often elegant approaches have been proposed to minimize the noise sensitivity [2, 9], for instance by weighting with the estimated DIC uncertainty [24] or with virtual fields [25-28]. However, all these approaches use the measured displacement (or post-processed strain) field, and not the actually acquired images, as a starting point, i.e. there is no direct transfer of information from images to properties and the identification process works unidirectionally only.

Ideally, steps $(i)$ and (ii) should be combined in a single inverse parameter identification procedure that enables for bidirectional information transfers and fully exploits the problem kinematics. The constitutive model contains the mechanically-admissible kinematic fields [29]. However, the conventional two-step approach separates the constitutive model from the DIC problem.

Recently, methods have appeared that directly relate the constitutive model to the experimentally obtained images, so-called Integrated DIC (I-DIC) algorithms. The main idea is to limit the image registration process to the kinematically admissible displacement modes, which are described by a geometrical and constitutive model, thereby directly solving for the unknown material parameters [30]. Several successful applications of the method have been published. The first series of publications applies I-DIC by using closed-form solutions to describe the kinematics of the experiments [30-33]. The second series applies I-DIC by using an approximated method (e.g. FEM) to obtain the sensitivity to the displacement field as a function of the unknown material parameters [29, 34-36]. This paper falls in the latter category, where a numerical method (i.e. FEM) will be used to approximate the required sensitivity fields to perform the direct one-step identification. In most papers discussing IDIC, the identification is still performed incrementally. Additionally, none of the previous papers challenge the method in cases with severe nonlinearity. In this paper a one-step I-DIC 


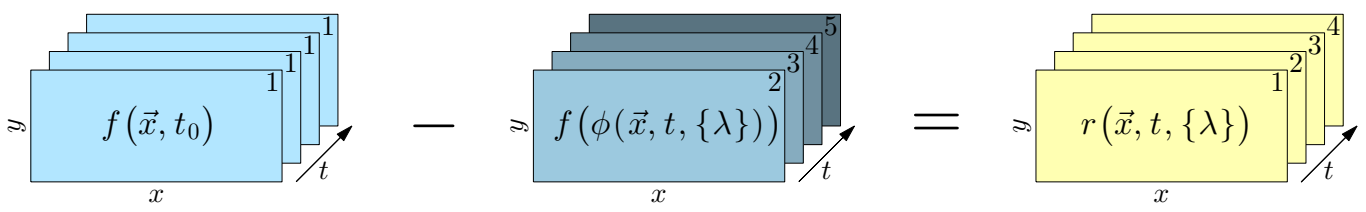

Figure 1. Schematic representation of the time-resolved brightness conservation, as written in Equation (1)

approach is presented that makes full use of the mechanics known from the constitutive model, while allowing the inclusion of all experimental data like images and force measurements in a single optimization problem. The method enables for a bi-directional information transfer where the noisy experimental data are regularized by the numerical model, while identifying the model parameters.

The paper is structured as follows. Section 2 treats the mathematical framework of the Integrated DIC approach, including the extensions of the framework to incorporate time integration and virtual boundaries. The potential of the approach is demonstrated in Sections 3 and 4 by two challenging examples, i.e. identification of the history- and ratedependent constitutive behavior of a glassy polymer (with 10 parameters) in an experiment exhibiting localization, and the parameter identification for two joined materials in a structured sample loaded by a bulge test setup ( 5 parameters). Finally, a discussion of the strengths and weaknesses of the methodology is given in Section 5 .

\section{METHODS}

\subsection{Time integrated DIC}

As with any DIC algorithm, the method starts off with assuming that the captured images contain a pattern that passively follows the material underneath. Therefore, the brightness of one material point is constant in all images, which is known as the brightness conservation [37]. However, with the goal of creating a single minimization process that includes all data, the brightness conservation equation is written to include all images, following the same reasoning as proposed by Besnard et al. [38]

$$
r_{1}(\vec{x}, t,\{\lambda\})=f\left(\vec{x}, t_{0}\right)-f(\phi(\vec{x}, t), t), \quad \phi(\vec{x}, t)=\vec{x}+\vec{u}(\vec{x}, t) .
$$

where, $f\left(\vec{x}, t_{0}\right)$ is the reference image, at the reference coordinates $\vec{x}$, and $f(\phi(\vec{x}, t), t)$ are all consecutive images evaluated at the deformed coordinates $\phi(\vec{x}, t)$. The residual field $r_{1}$ will reduce to the acquisition noise if the correct deformation field $\vec{u}(\vec{x}, t)$ is applied.

The above equation is the equivalent of stacking all images together into one large (3D) matrix. The residual is formed by deforming each image back to the initial configuration (i.e. at $t=t_{0}$ ) by applying the mapping function for the corresponding time and comparing it to the initial frame (Figure 1). Note that for the computation of $r_{1}$, the gray values at the deformed coordinates are typically not at integer pixel locations, and thus require pixel interpolation, which is here performed by resorting to cubic B-splines [39].

To remedy the ill-posedness inherent to DIC algorithms, the displacement field is parameterized with a limited number of unknowns

$$
\vec{u}^{*}(\vec{x}, t,\{\lambda\}) \approx \vec{u}(\vec{x}, t),
$$

where $\vec{u}^{*}$ is the approximated displacement field, and $\{\lambda\}$ is a vector with $n$ degrees of freedom $\{\lambda\}=\left[\lambda_{1}, \lambda_{2}, \lambda_{i}, \ldots, \lambda_{n}\right]^{t}$. From this point onward, the superscripted asterisk is omitted since the dependence of a field on $\{\lambda\}$ indicates that it is an approximated quantity. 
The residual field is now expressed as

$$
r_{1}(\vec{x}, t,\{\lambda\})=f\left(\vec{x}, t_{0}\right)-f(\phi(\vec{x}, t,\{\lambda\}), t), \quad \phi(\vec{x}, t,\{\lambda\})=\vec{x}+\vec{u}(\vec{x}, t,\{\lambda\}),
$$

where the deformation mapping is now a function of the degrees of freedom. The solution $\{\lambda\}$ is obtained from the quadratic residual norm, $\Phi_{1}$,

$$
\{\lambda\}=\underset{\{\lambda\}}{\operatorname{Argmin}}\left(\Phi_{1}(\{\lambda\})\right) \quad \Phi_{1}(\{\lambda\})=\int_{\tau} \int_{\Omega}\left[r_{1}(\vec{x}, t,\{\lambda\})\right]^{2} \mathrm{~d} \vec{x} \mathrm{~d} t,
$$

where $\Omega$ is the region of interest (ROI) over which the residual field is minimized, which can also be a 3D volume if Digital Volume Correlation is considered [40,41], and $\tau$ the corresponding time domain of interest that adds another dimension to $r_{1}$. The solution for the optimal degrees of freedom is a nonlinear problem, which is linearized and solved for iteratively, for instance, with a Newton-Raphson scheme [15, 37]. The linearized system of equations is usually written in matrix form as

$$
[\mathbf{M}] \delta\{\lambda\}=\{\mathbf{b}\},
$$

where $\delta\{\lambda\}$ is the iterative update of the degrees of freedom $\{\lambda\}^{(k+1)}=\{\lambda\}^{(k)}+\delta\{\lambda\},\{\lambda\}^{(k)}$ is initialized with an initial guess $\{\lambda\}_{0}$. The components of the DIC matrix $[\mathbf{M}]$ and the right hand side member $\{\mathbf{b}\}$ read

$$
\begin{aligned}
M_{i j} & =\int_{\tau} \int_{\Omega}\left(\left(\vec{\nabla} f \cdot \vec{\varphi}_{i}\right)\left(\vec{\nabla} f \cdot \vec{\varphi}_{j}\right)\right) \mathrm{d} \vec{x} \mathrm{~d} t, \\
b_{j} & =\int_{\tau} \int_{\Omega}\left(\left(\vec{\nabla} f \cdot \vec{\varphi}_{j}\right) r_{1}\right) \mathrm{d} \vec{x} \mathrm{~d} t,
\end{aligned}
$$

where, $\vec{\nabla} f$ is the applied image gradient, and $\vec{\varphi}_{i}$ are the basis functions defined as

$$
\vec{\varphi}_{i}(\vec{x}, t,\{\lambda\})=\frac{\partial u(\vec{x}, t,\{\lambda\})}{\partial \lambda_{i}} .
$$

The derivative of the displacement field with respect to a degree of freedom represents the sensitivity field to that degree of freedom. For some particular cases, it is possible to formulate closed-form relationships between the unknown material parameters and the displacement field [31-33]. For cases where this is not feasible, it is possible to approximate these sensitivity maps by numerical differentiation of the simulated displacement fields $[29,34,35]$. To this end, the reference displacement field, obtained with the current degrees of freedom $\{\lambda\}^{(k)}$ (i.e. from an FE simulation), is perturbed $n$ times, each time perturbing only one degree of freedom with a small perturbation $\epsilon$

$$
\vec{\varphi}_{i}\left(\vec{x}, t, \lambda^{(k)}\right) \approx \frac{\vec{u}^{k}\left(\vec{x}, t,(1+\epsilon) \lambda_{i}^{(k)}\right)-\vec{u}^{k}\left(\vec{x}, t,\{\lambda\}^{(k)}\right)}{\epsilon \lambda_{i}^{(k)}} .
$$

To obtain the sensitivity fields at the pixel coordinates (i.e. $\vec{x}$ ), the FE shape functions are applied to interpolate the nodal values on the pixel coordinates. Similarly the FE reference displacement field is interpolated on the pixel locations and applied to compute the residual

$$
r_{1}^{k}\left(\vec{x}, t,\{\lambda\}^{(k)}\right)=f\left(\vec{x}, t_{0}\right)-f\left(\phi\left(\vec{x}, t,\{\lambda\}^{(k)}\right), t\right), \quad \phi\left(\vec{x}, t,\{\lambda\}^{(k)}\right)=\vec{x}+\vec{u}^{(k)}\left(\vec{x}, t,\{\lambda\}^{(k)}\right)
$$

All ingredients required to solve for the update of the degrees of freedom are in place. The resulting iterative procedure is run until convergence is met. In this paper, the convergence criterion is based on the $L^{2}$-norm of the right hand side member

$$
\|\{\mathbf{b}\}\|<10^{-5},
$$


However, it is also possible to define a convergence criterion in terms of the 'length' of the iterative update $\|\delta\{\lambda\}\|[34]$.

Using the sensitivity maps as the basis functions of the DIC algorithm is an important feature of the proposed method. It provides a direct connection between the acquired images and the unknown material parameters, which creates a bidirectional information transfer between images and mechanical properties, allowing for direct material parameter identification while providing strong regularization of the DIC algorithm, thereby ensuring optimal accuracy and noise robustness. The simulation method required for Equation (9) is not limited to FE nor to the imaged surface. Areas that lie outside of the imaged field of view can be included in the simulation. This allows for the identification of parameters that influence the imaged surface but are defined from outside the field of view, or from deeper in the material (3D) [34].

\subsection{Additional experimental data}

In a typical experiment, additional data are measured, for instance, the displacement or the force at the clamps of the tensile stage or images recorded by a second camera. To clarify the incorporation of supplementary experimental data in the proposed I-DIC framework, the measured force is next used as an example.

For some cases, it is possible to include the measured force by load-controlled FE simulations with the recorded force history (see Section 4). However, this is not feasible for structurally softening samples, which require displacement control because of the snap-through response (see Section 3). To include the experimentally measured force in the minimization procedure, a second residual is defined,

$$
r_{2}(t,\{\lambda\})=F_{\exp }(t)-F_{\text {sim }}(t,\{\lambda\})
$$

where $F_{\text {exp }}$ is the experimentally measured force as a function of time, and $F_{\text {sim }}$ the corresponding simulated reaction force, which depends on the sought degrees of freedom. The force residual is combined with the brightness residual (Equation (10)) to form a single residual such that only one solution for the parameters $\lambda$ is obtained

$$
\Phi(\{\lambda\})=(1-\alpha) \frac{1}{\beta_{1}} \Phi_{1}(\{\lambda\})+\alpha \frac{1}{\beta_{2}} \Phi_{2}(\{\lambda\}), \quad \Phi_{2}(\{\lambda\})=\int_{\tau}\left(r_{2}(t,\{\lambda\})\right)^{2} \mathrm{~d} t .
$$

The parameters $\beta_{i}$ are normalization constants, defined as the $L^{2}$-norm of their respective matrix $[\mathbf{M}]_{i}$, and $\alpha \in[0,1]$ allows to shift weight from one functional to the other. Typically, more weight is put on the optical part, i.e. $\alpha=0.01$ [34], which is also done in this paper. However, a recent paper discusses a method for choosing $\alpha$ based on estimates of the accuracy of the data for each contribution, i.e. the estimated acquisition noise [36].

The two residuals are independent of each other, and therefore can be linearized independently, resulting in the following linear system of equations, which is applied iteratively

$$
\begin{aligned}
{[\mathbf{M}] \delta\{\lambda\} } & =\{\mathbf{b}\} \\
\left((1-\alpha) \frac{1}{\beta_{1}}[\mathbf{M}]_{1}+\alpha \frac{1}{\beta_{2}}[\mathbf{M}]_{2}\right) \delta\{\lambda\} & =\left((1-\alpha) \frac{1}{\beta_{1}}\{\mathbf{b}\}_{1}+\alpha \frac{1}{\beta_{2}}\{\mathbf{b}\}_{2}\right),
\end{aligned}
$$

The optical part of this linearized system, $[\mathbf{M}]_{1}$ and $\{\mathbf{b}\}_{1}$, is exactly the same as above defined (Equations (6) and (7)). The force part of the system results from the Newton-Raphson scheme

$$
\begin{aligned}
M_{2(i j)} & =\int_{\tau}\left(\rho_{i} \rho_{j}\right) \mathrm{d} t, \\
b_{2(j)} & =\int_{\tau}\left(\rho_{j} r_{2}\right) \mathrm{d} t,
\end{aligned}
$$




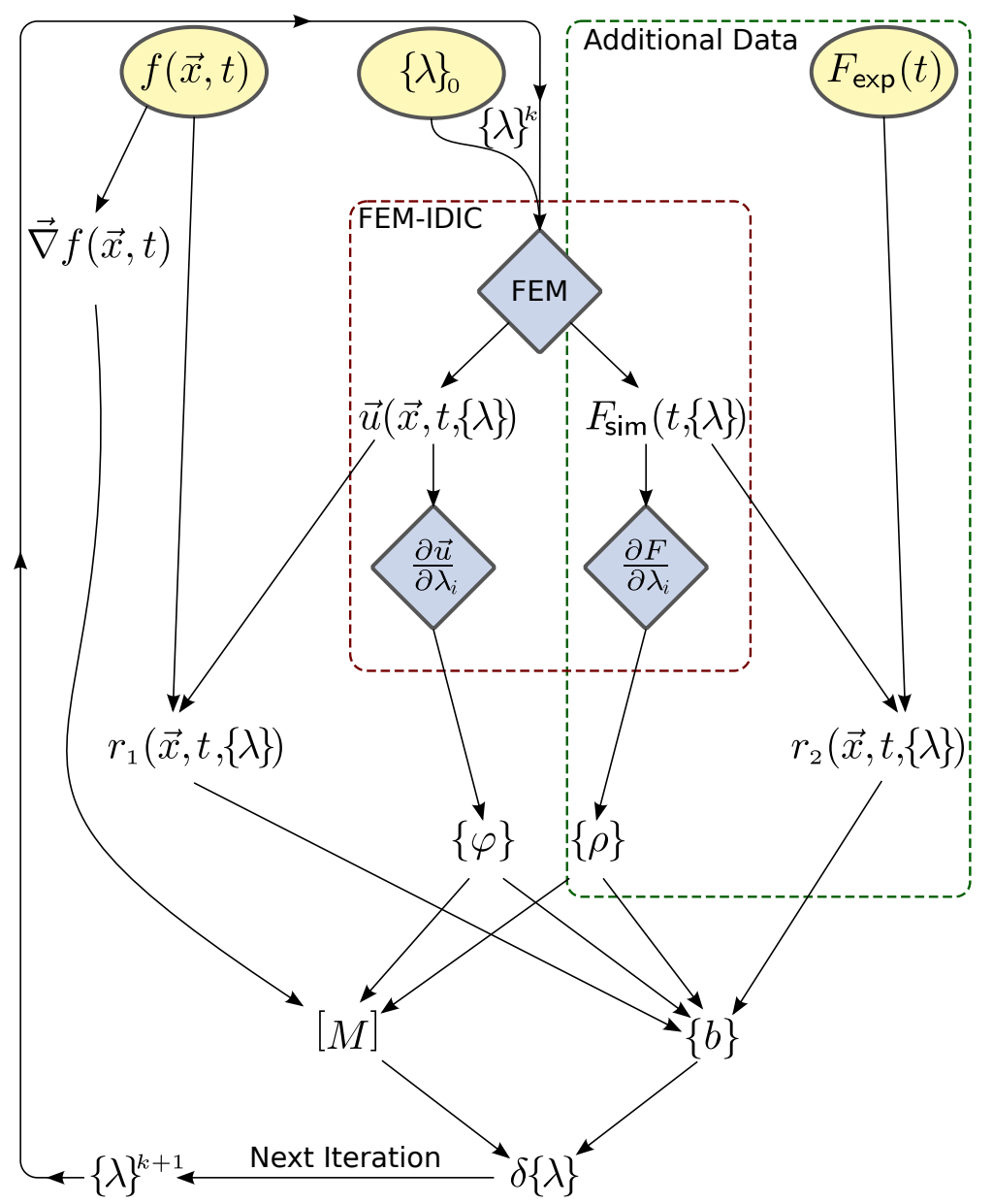

Figure 2. Block diagram of the proposed I-DIC framework

where $\{\rho\}(t,\{\lambda\})$ are force sensitivities complementary to the displacement sensitivity fields $[\varphi](\vec{x}, t,\{\lambda\})$. These fields are obtained through numerical differentiation of the FE simulations

$$
\rho_{i}(t,\{\lambda\}) \approx \frac{F^{(k)}\left(t,(1+\epsilon) \lambda_{i}^{(k)}\right)-F^{(k)}\left(t,\{\lambda\}^{(k)}\right)}{\epsilon \lambda_{i}^{(k)}} .
$$

Any additional experimental data can be added to the minimization problem in this manner, as long as it has a numerical counterpart. For instance, measured temperature fields can be compared to thermo-mechanical FE results.

This part completes the proposed FEM-based I-DIC method. The flow of information in the proposed method is summarized as a block diagram in Figure 2. The implementation does not substantially differ from a typical global DIC implementation. The major difference is that the basis functions are not chosen in advance, since they are the sensitivity fields obtained by numerical differentiation of the FE simulations. In addition, since it is almost trivial to compute time-resolved sensitivity fields from the FE data, it is natural to formulate a timeresolved residual image, which allows for robust identification of history-dependent parameters. Additionally, the experimental force needs to be included in the method to obtain absolute values for the stiffness, otherwise only stiffness ratios can be determined. 


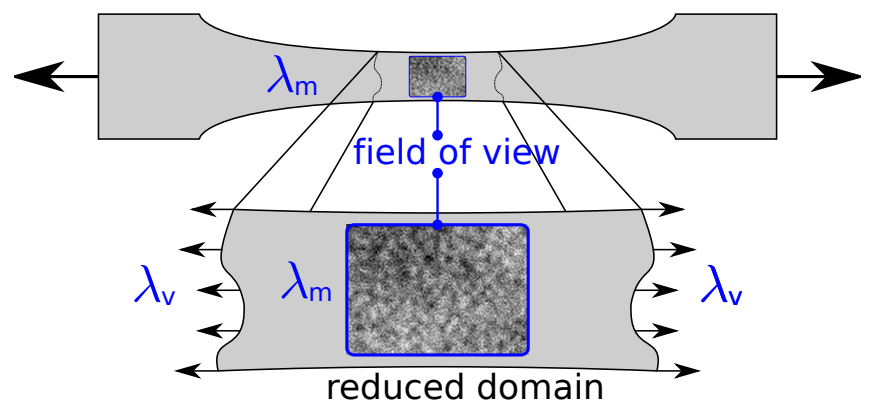

Figure 3. Additional degrees of freedom $\{\lambda\}_{v}$ (complementary to the material degrees of freedom $\left.\{\lambda\}_{m}\right)$ applied to create a virtual boundary, effectively reducing the FE domain

\subsection{Additional degrees of freedom}

Up to this point, the discussed degrees of freedom were the unknown material parameters. However, the proposed method is not limited to identifying material parameters. Similarly as what is possible with FEMU or other inverse identification methods, with the proposed I-DIC method any defined parameter, which has an influence on the minimization problem, can be identified. One possible use of additional dof is to provide virtual boundaries [42]. Typically, the captured field of view does not contain the entire sample. However, to apply the correct boundary conditions, it would seem most logical to model the entire sample in the FE simulation. However, a more elegant choice may be to apply a parameterized boundary located intermediately between the real sample boundary and the field of view, and add this boundary as parameters to the $d o f$. In this way, the simulated domain can be greatly reduced, which reduces the computational cost per simulation (Fig. 3). Other examples include, identification of the sample geometry, calibration of camera parameters for one or multiple cameras [43].

The process of adding an extra degree of freedom is as straight forward as defining the parameter and including it in the FEM simulation. Consequently by the evaluation of Equation (9) the corresponding sensitivity field will naturally follow. In this manner, an arbitrary set of $d o f$ can be added to the system, although not without consequences. Additional dof may degrade the conditioning or even cause non-uniqueness of the solution. However, this can be predicted by evaluating the conditioning of $[\mathbf{M}]$. Moreover, an extra FE simulation has to be performed, for each I-DIC iteration, for each additional degree of freedom, increasing the computational cost.

\section{POLYCARBONATE CHARACTERIZATION}

As a first proof-of-principle example the constitutive properties of PolyCarbonate (PC) are identified. PC is a glassy polymer that is rate dependent (i.e. viscoelastic) and history dependent. The polymer chains tend to diffuse to a low energy state that compacts the material, thereby increasing the yield stress. Material flow induces rejuvenation, which has a reverse effect on the yield stress, causing flow-induced softening [44, 45]. It is this softening behavior combined with the stress- and temperature-dependent viscosity that makes this material a challenging test case for the proposed I-DIC method.

\subsection{Glassy polymer model}

A particular constitutive model, which is well suited for modeling PC is the so-called Eindhoven Glassy Polymer (EGP) model, which is actively being developed [47, 48]. For details about the model the reader is referred to [49]. To introduce the material parameters the constitutive model is briefly discussed in Appendix B. 


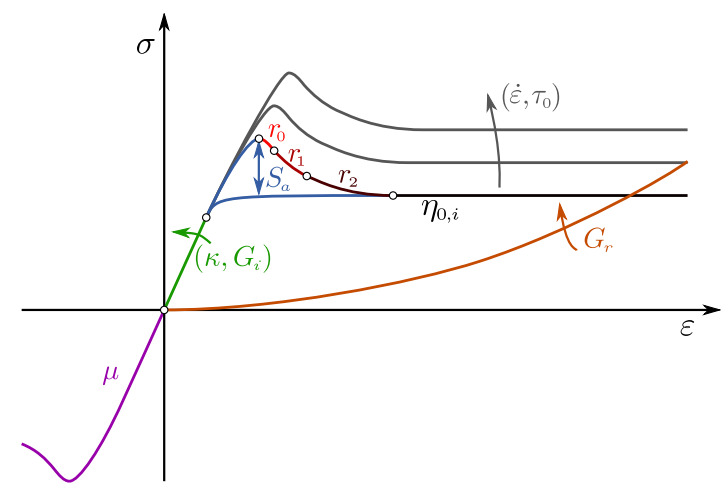

(a)

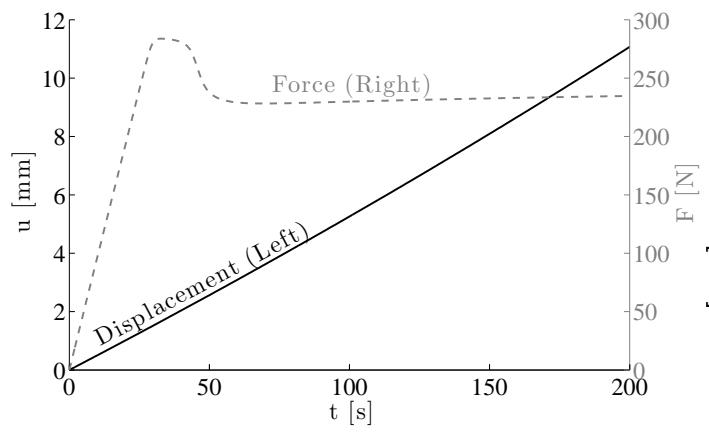

(c)

\begin{tabular}{llcl}
\hline$\lambda_{1}$ & $G_{r}$ & 26 & {$[\mathrm{MPa}]$} \\
$\lambda_{2}$ & $S_{a}$ & 27 & {$[-]$} \\
$\lambda_{3}$ & $r_{0}$ & 0.965 & {$[-]$} \\
$\lambda_{4}$ & $r_{1}$ & -3 & {$[-]$} \\
$\lambda_{5}$ & $r_{2}$ & 50 & {$[-]$} \\
$\lambda_{6}$ & $\tau_{0}$ & 0.7 & {$[\mathrm{MPa}]$} \\
$\lambda_{7}$ & $\mu$ & 0.08 & {$[-]$} \\
$\lambda_{8}$ & $\kappa$ & 3.75 & {$[\mathrm{GPa}]$} \\
$\lambda_{9}$ & $G_{1}$ & 634 & {$[\mathrm{MPa}]$} \\
$\lambda_{10}$ & $\eta_{0,1}$ & $1.47 \cdot 10^{11}$ & {$[\mathrm{MPa} \cdot \mathrm{s}]$} \\
\hline
\end{tabular}

(b)
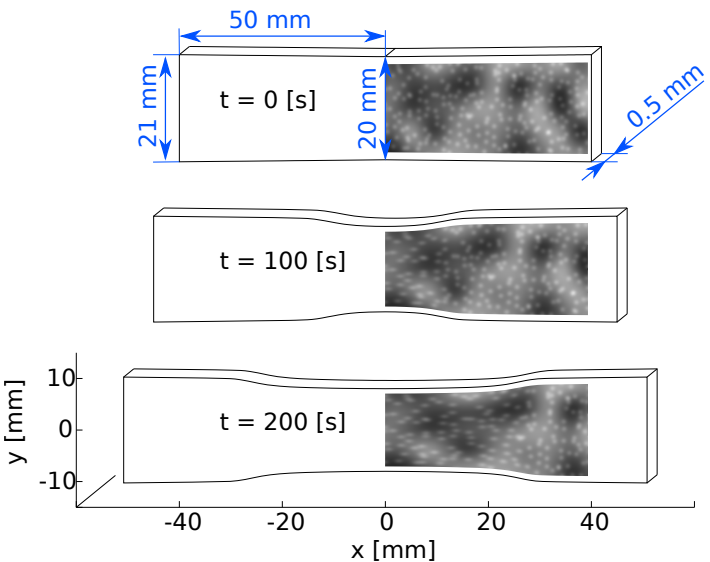

(d)

Figure 4. (a) Sketch of the influence of the various EGP parameters on the stress-strain response. (b) Reference single mode EGP parameters for the polycarbonate sample [46]. (c) Applied displacement boundary condition and measured force response. (d) Contour of the mesh in the undeformed, intermediate, and deformed states. The adopted DIC pattern is depicted in the mesh

The influence of the EGP constitutive parameters on the stress-strain response is illustrated in Figure 4a. The two contributions to the stress tensor $\boldsymbol{\sigma}_{s}$ and $\boldsymbol{\sigma}_{r}$ are shown separately, whereby the network contribution $\boldsymbol{\sigma}_{r}$ (shown in orange) is controlled by the neo-Hookean modulus $G_{r}$. The driving stress $\boldsymbol{\sigma}_{s}$ is controlled by the other 9 parameters. Five of the latter ones are typical non-Newtonian parameters, with $\kappa$ the bulk modulus, $G_{i}$ the intermolecular contribution to the shear modulus, $\eta_{0, i}$ the corresponding zero-viscosity and $\tau_{0}$ the characteristic stress. The remaining four parameters control aging and rejuvenation, where $S_{a}$ is the aging state parameter that depends on the history of the material, $r_{0}, r_{1}$ and $r_{2}$ describe the softening response. The state $S_{a}$ is particularly important since it is used to predict the product lifetime $[50,51]$.

\subsection{Virtual experiment}

To accurately identify all EGP parameters it is important that they are 'probed' during the experiment and influence the observed pattern. Because PC exhibits flow induced softening and strain hardening, a simple tensile experiment will form a neck that subsequently grows in a stable fashion. It is known that the necking geometry is sensitive to the aging and rejuvenation kinetics [45]. Moreover, the moving neck creates an area with a gradient in strain and strain rate. It is therefore assumed that a simple tensile experiment on a strip of PC contains sufficient information to identify all 10 EGP parameters.

To test the performance of the identification process it is important that the targeted parameters are known exactly beforehand, so that they can be compared to the obtained parameters. To this end, a virtual experiment is performed, where the images normally obtained by a camera are artificially generated by deforming a reference pattern with the 

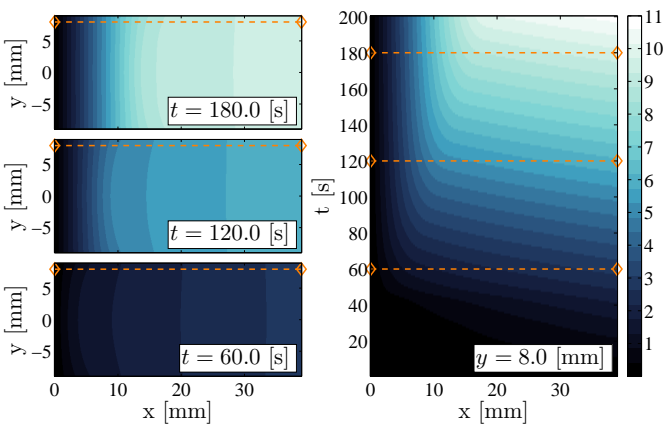

(a) $u_{x}$

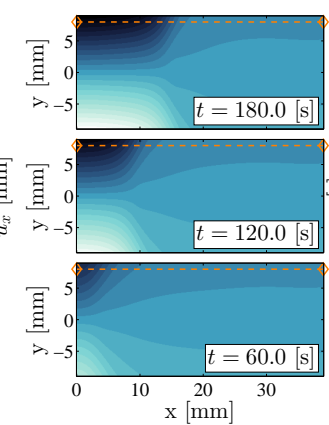

(b) $u_{y}$

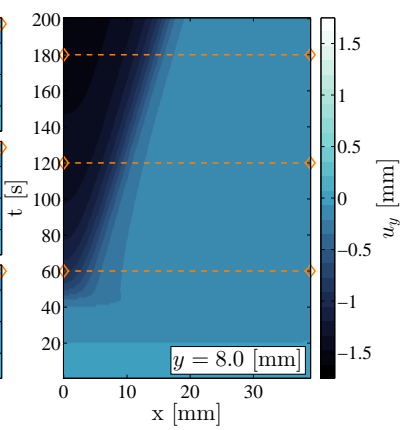

Figure 5. (a) $x$-component and (b) $y$-component of the displacement field for three time increments and for a single $y$-plane and all time increments.

displacement field obtained from a reference FE simulation. The virtual experiment enables a controlled addition of artificial measurement uncertainties such as acquisition noise, to evaluate noise robustness.

The applied tested sample is a PC strip $\left(100 \times 21 \times 0.5 \mathrm{~mm}^{3}\right)$ that tapers slightly towards the center to enforce that necking starts there. The applied EGP parameters are listed in Table $4 \mathrm{~b}$ and are typical for PC [46]. The sample is loaded in tension with a constant global strain rate of $\dot{\varepsilon}=0.001\left[\mathrm{~s}^{-1}\right]$ for 200 seconds, see Figure 4c. Because of the large strains occurring in the experiment, a relatively sparse pattern is applied, as shown in Figure 4d. The sample shape and pattern are shown in Figure $4 \mathrm{~d}$ for the initial, intermediate and final deformation states, clearly showing the necking phenomenon.

The FEM model applied in this virtual experiment is the same model as applied in the forward part of the identification routine (i.e. Equation (9)). Due to symmetry, only the right side of the sample is modeled (i.e. $0 \leq x \leq 50 \mathrm{~mm}$ ), using 8-noded tri-linear hexahedron (brick) elements resulting in a mesh with 4719 nodes connected by 2881 elements, where only one element over the thickness (z-direction) is used. The simulation is performed in load-control over 2000 time increments of $0.1 \mathrm{~s}$, of which 200 increments at $1 \mathrm{~s}$ intervals are stored and used to generate 8-bit images that span a field of view of approximately $39.1 \times 18.0 \mathrm{~mm}^{2}$, with a pixel size of $93.1 \mathrm{\mu m}$. See appendix A for details regarding the computational cost.

\subsection{Parameter sensitivity}

Figure 5 shows the $x$ - and $y$-component of the displacement for three time increments (i.e. at 60,120 , and $200 \mathrm{~s}$ ). Next to the three time-slices, a $y$-slice is presented that shows the respective component of the displacement vector for all time steps at a single $y$-location, i.e. $y=8 \mathrm{~mm}$. This $x$ - $t$ representation is useful since the variations along the $y$-direction are typically obvious. Let us emphasize that the onset of necking and stable neck propagation is visible as a line feature at an angle in the $x$-t plane starting at approximately $50 \mathrm{~s}$.

The basis functions applied in the proposed I-DIC method are the sensitivity fields (i.e. Equation (9)) of the displacement field with respect to the unknowns, which for this example are the 10 EGP parameters (Table $4 \mathrm{~b}$ ). The sensitivity fields can be computed before the experiment is performed and are at that state already a useful tool to diagnose the sensitivity of the parameters for the planned experiment. Each computed sensitivity field $\vec{\varphi}_{i}$ is a 3D volume of $2 \mathrm{D}$ vectors as for the above discussed displacement fields. Additionally, there is a force sensitivity vector $\rho_{i}$, which is a $1 \mathrm{D}$ scalar field. Both vector components of $\vec{\varphi}_{i}$ and the force sensitivity $\rho_{i}$ are connected to a single degree of freedom.

For the ease of plotting, all sensitivity fields are scaled with their respective (absolute) maximum value of the $x$-component, (i.e. $\hat{\lambda}_{i}$ ). Figure 6 shows a single $x$-t-plane (at $y=8 \mathrm{~mm}$ ) for all 10 scaled sensitivity fields. Degrees of freedom that have limited impact on the force 

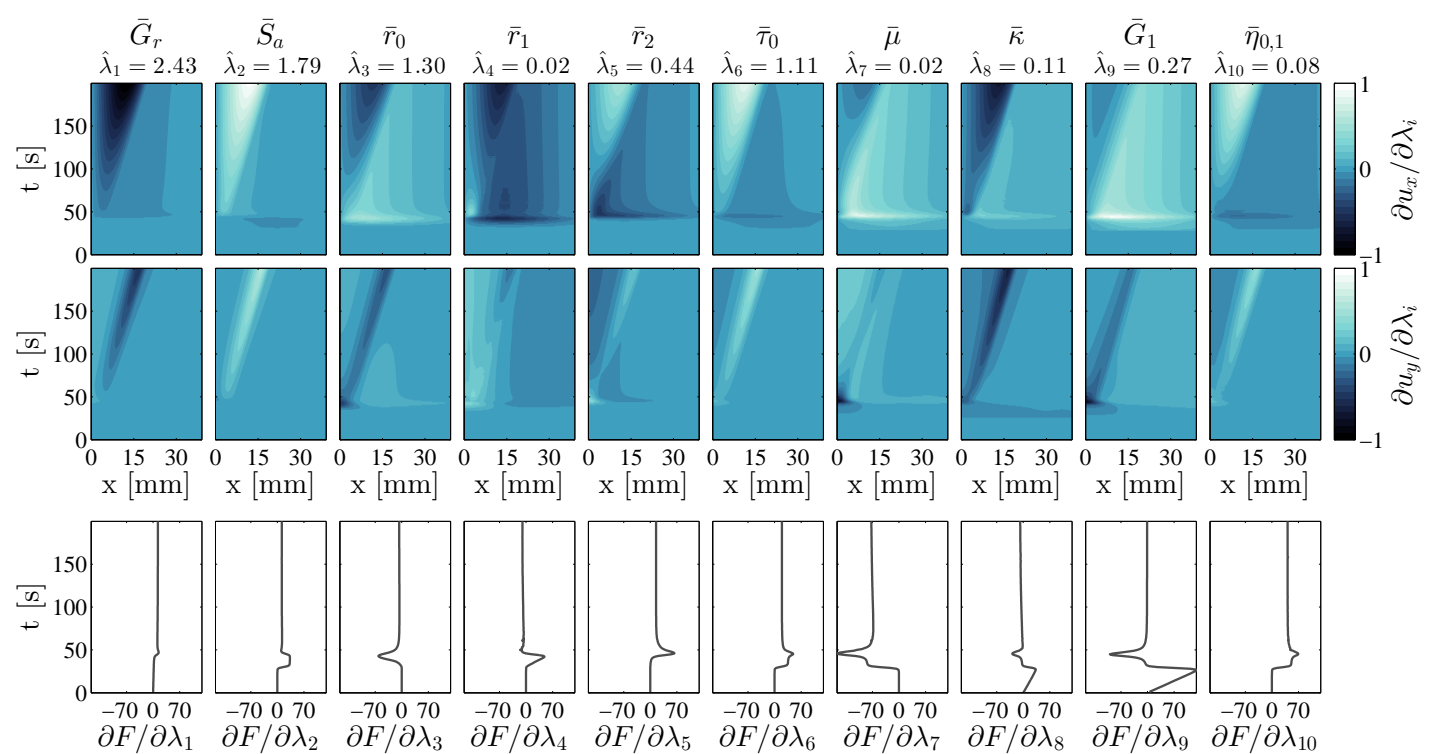

Figure 6. The scaled sensitivity maps (or basis functions) for all 10 unknown material parameters, for a single $y$-plane $(y=8.0 \mathrm{~mm})$. The three figures in one dof column combine to one sensitivity field. The values of the scaling parameters $\hat{\lambda}_{i}$ are shown on top of the respective sensitivity maps

or displacement field will have low values in the sensitivity map, which is most visible in the value of the respective $\hat{\lambda}_{i}$, shown at the top of each sensitivity map. In this case, $r_{1}$ has the lowest displacement sensitivity, followed by $\mu$ and $\eta_{0,1}$.

The reduced sensitivity for these degrees of freedom is expected. The $r_{1}$ parameter only influences a limited regime in the stress-strain curve, see Figure 4a. Consequently, only a small volume of material is affected by $r_{1}$ at each time increment. The $\mu$ parameter controls the pressure dependence, and for this particular experiment it is unlikely that large differences in pressure occur. Sensitivity for the zero-viscosity $\eta_{0,1}$ requires large differences in strain rate. The area in the neck is straining at a different rate compared with that in front of the neck. However, the expected strain rate differences are small compared to what is typically applied to quantify the zero-viscosity. As a consequence to the small variations in strain rate, also a low sensitivity is expected in the characteristic stress $\tau_{0}$. However, this parameter also controls the onset of flow, to which this experiment is sensitive.

As a proof-of-principle experiment the identification procedure was initiated with an initial guess for each parameter equal to $110 \%$ of their reference value. After 30 iterations, convergence is reached, and values for all 10 EGP parameters have been identified. To evaluate the quality of the identification, first the residual field (stack) is analyzed. Figure 7 shows the residual from before (i.e. using the initial guess) and after the I-DIC procedure is run. Since this (virtual) procedure contained no measurement noise, the residual field is reduced to almost zero, the only visible features are due to sub-pixel interpolations.

The analysis of the residual is important and always possible. However, since a virtual experiment method is applied here, the reference displacement field is known as well. The difference between the obtained displacement field and the reference displacement field is analyzed next, which is defined as

$$
\begin{aligned}
& \mathcal{E}_{u_{x}}=\left[u_{x}\right]_{\mathrm{ref}}-\left[u_{x}\right]_{\mathrm{idic}}, \\
& \mathcal{E}_{u_{y}}=\left[u_{y}\right]_{\mathrm{ref}}-\left[u_{y}\right]_{\mathrm{idic}} .
\end{aligned}
$$

Both error fields are shown in Fig. 8. The largest displacement error is in the $x$-displacement, occurring at the onset of necking and equals $0.3 \mu \mathrm{m}$, which is $0.3 \%$ of the pixel size (i.e. $93 \mu \mathrm{m}$.). This is well below the typical $10^{-2}$-pixel resolution typically obtained in regular 


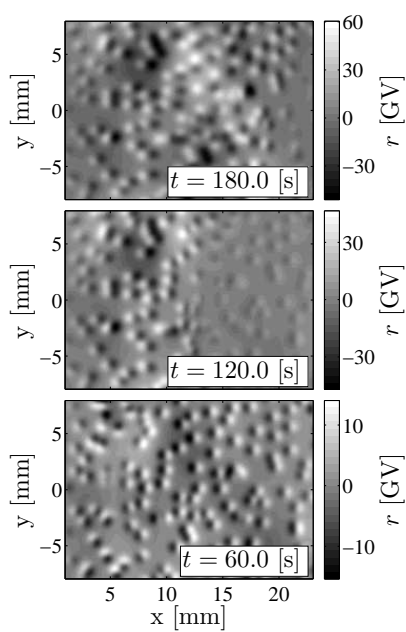

(a)

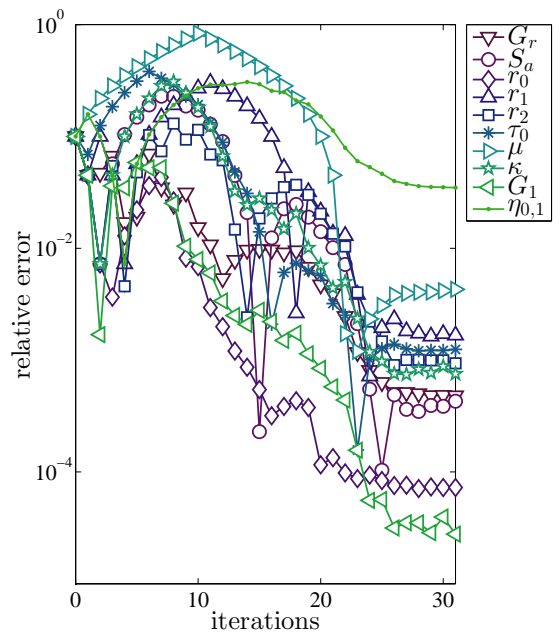

(b)

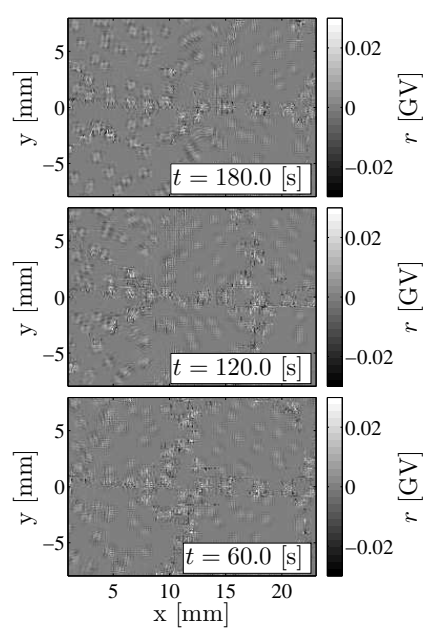

(c)

Figure 7. (a) Three residual images taken at the start (i.e. based on the initial guess of $110 \%$ relative to the reference). (b) Convergence behavior of the I-DIC routine, identifying all 10 EGP material parameters. (c) Three final residual images (i.e. from the final iteration). Note the difference in scale bar values between the initial and the final residual
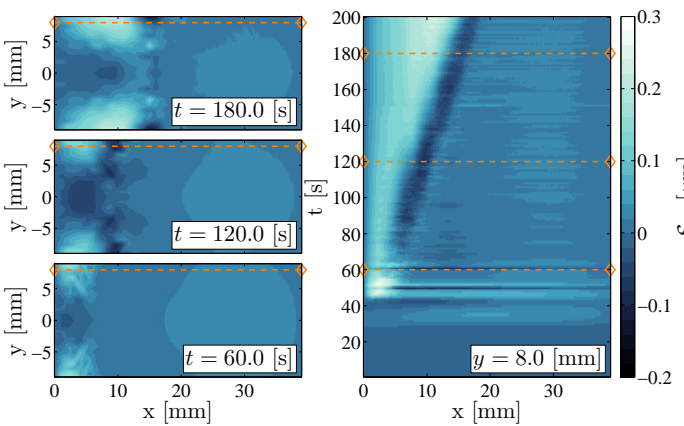

(a)

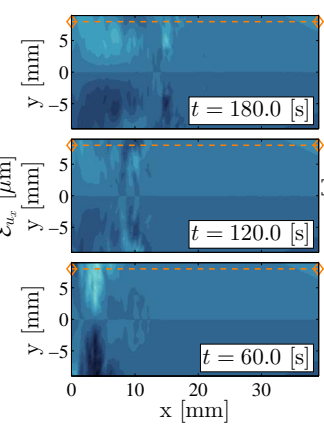

(b)

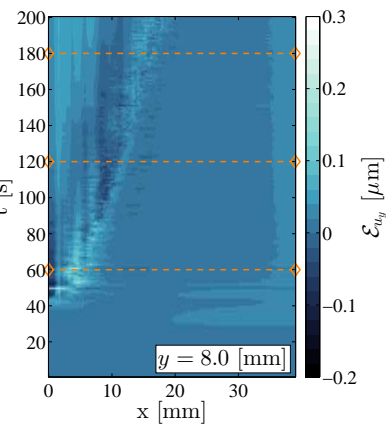

Figure 8. (a) $x$-component and (b) $y$-component of the displacement error field for three time increments, for a single $y$-plane and all time increments.

DIC algorithms [21]. This good accuracy in the displacement field translates into the obtained parameters as shown in Table I. All obtained parameters are within $1 \%$ of the expected values, except for the zero-viscosity $\eta_{0,1}$, which stagnates at $3.5 \%$ error. The error in the material parameters highlights the stringent demands on the displacement accuracy.

The presented test case is devoid of model error, which is possible since it is a virtual test. However, in real experiments, there will always be some model error (large or small, depending on the quality of the constitutive model). Consequently, in the presence of significant model error, the displacement field obtained with I-DIC will not coincide with one measured with an un-regularized technique such as DIC. In such a situation the difference between the I-DIC and DIC displacement fields is a form of inaccuracy. However, it is questionable if this should be called measurement inaccuracy. If a method like FEMU were applied, the displacement field obtained after convergence would neither coincide with the DIC displacement field, and would be similarly inaccurate. If there is a significant model error, the presented I-DIC method will give the closest possible description of the experiment allowable within the constitutive framework (see also Section 4.5). 
Table I. The identified EGP parameters (the initial guess for each degree of freedom was $10 \%$ off the expected value)

\begin{tabular}{cccccccccc}
\hline$\lambda_{1}$ & $\lambda_{2}$ & $\lambda_{3}$ & $\lambda_{4}$ & $\lambda_{5}$ & $\lambda_{6}$ & $\lambda_{7}$ & $\lambda_{8}$ & $\lambda_{9}$ & $\lambda_{10}$ \\
$G_{r}$ & $S_{a}$ & $r_{0}$ & $r_{1}$ & $r_{2}$ & $\tau_{0}$ & $\mu$ & $\kappa$ & $G_{1}$ & $\left.\begin{array}{c}\eta_{0,1} \\
{[-]}\end{array}\right]$ \\
{$[\mathrm{MPa}]$} & {$[-]$} & {$[-]$} & {$[-]$} & {$[-]$} & {$[\mathrm{MPa}]$} & {$[-]$} & {$[\mathrm{GPa}]$} & {$[\mathrm{MPa}]$} & {$[\mathrm{MPa} \cdot \mathrm{s}]$} \\
\hline 26.01 & 26.99 & 0.965 & 4.99 & -2.99 & 0.70 & 0.080 & 3.753 & 6.34 & $1.42 \cdot 10^{11}$ \\
$0.05 \%$ & $-0.04 \%$ & $0.01 \%$ & $-0.17 \%$ & $-0.09 \%$ & $0.12 \%$ & $0.44 \%$ & $0.07 \%$ & $-0.003 \%$ & $-3.48 \%$ \\
\hline
\end{tabular}

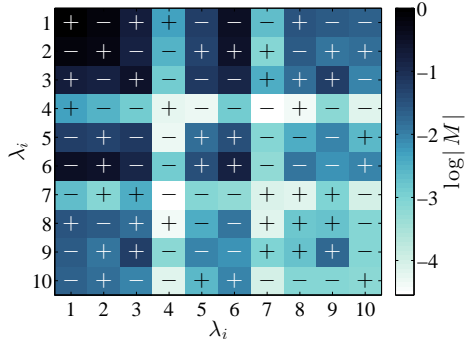

(a)

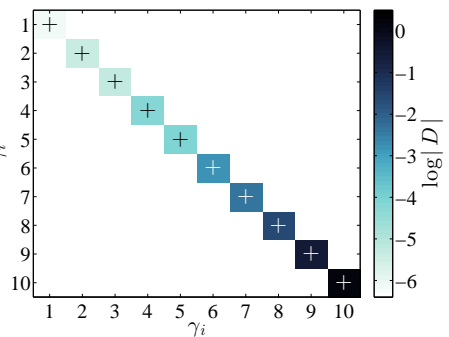

(b)

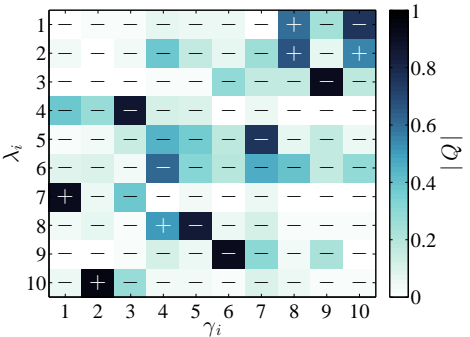

(c)

Figure 9. Graphical representation of the DIC matrix $[\mathbf{M}]$ and the eigenvalue matrix $[\mathbf{D}]$ and the eigenvector matrix $[\mathbf{Q}]$. The sign of the matrix value is displayed by the symbol in the center of each matrix component.

As predicted by the analyses of the sensitivity fields, the three degrees of freedom with the least sensitivity are also the three where the identification is the least accurate. This sensitivity analysis is only based on the numerical results and does not include the image gradient. The combined effect of the sensitivity fields and the pattern gradient is best evaluated by analyzing the DIC matrix [M] , as discussed in [21]. The DIC matrix is presented graphically in Figure 9a, where high values of $[\mathbf{M}]$ indicate a high sensitivity. From Figure 9a it is immediately clear that the rows and columns belonging to $\lambda_{4}$ and $\lambda_{7}$ are the least sensitive. To show this more clearly, a spectral decomposition of $[\mathbf{M}]$ is applied

$$
[\mathbf{M}]=[\mathbf{Q}][\mathbf{D}][\mathbf{Q}]^{-1}
$$

where $[\mathbf{D}]$ is a diagonal matrix with the eigenvalues $\gamma_{i}$ of $[\mathbf{M}]$ on the diagonal, shown in Figure $9 \mathrm{~b} .[\mathbf{Q}]$ is the corresponding eigenvector matrix where each column represents an eigenvector associated with the respective eigenvalue, shown in Figure 9c. Consider the three eigenvalues with the lowest levels, corresponding to the three left most columns in Figure 9c. Since these three eigenvalues have relatively low values, their corresponding eigenvectors represent combinations of $d o f$ (directions in the solution space) to which the method is particularly insensitive. These three vectors are mostly dominant in $\lambda_{7}=\mu, \lambda_{10}=\eta_{0,1}$ and $\lambda_{4}=r_{1}$. Additionally, the remaining eigenvectors show inferior dependence on those three $d o f$. This leads to the conclusion that not only $\lambda_{4}$ and $\lambda_{7}$ are suspected to be insensitive, as visible from $[\mathbf{M}]$, but also $\lambda_{10}$, which is consistent with the obtained accuracy as shown in Table I. This result shows that, for this case, the contrast in accuracy could have been assessed beforehand.

\subsection{Robustness with respect to the initial guess}

Inverse methods are often compromised by non-uniqueness or non-convexity problems [6, 7]. The applied Newton-Raphson minimization scheme is known for its excellent convergence properties when close to the solution. However, it may converge to a local minimum, or not converge at all, when initiated too far from the solution. To test the robustness against poor initial guesses, the discussed example case was re-initialized at various distances from the 


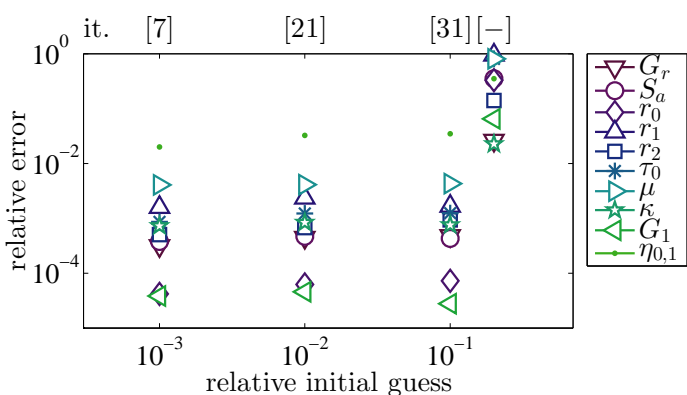

(a)

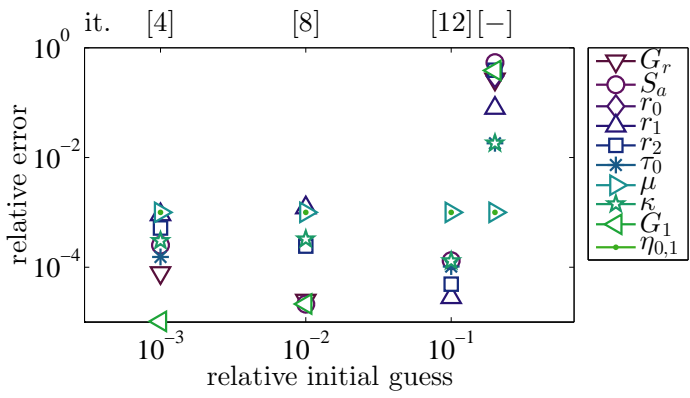

(c)

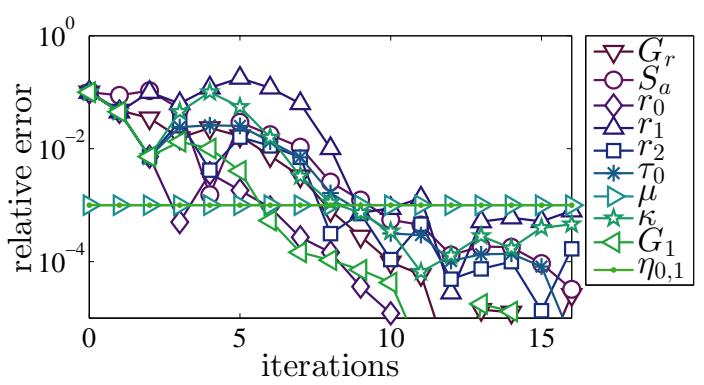

(b)

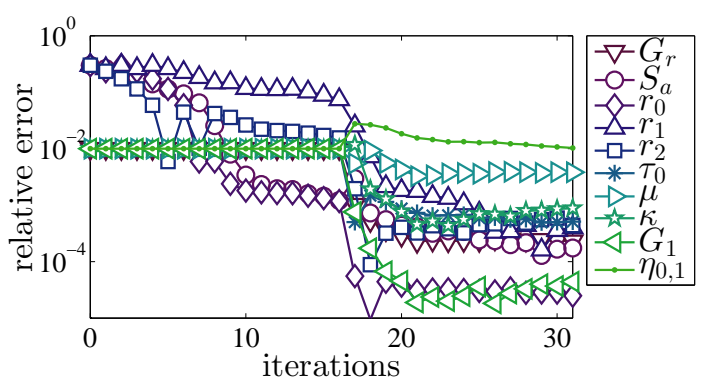

(d)

Figure 10. (a) Converged relative error for the identified parameters as a function of the relative initial guess, identifying all 10 EGP material parameters. (b) Convergence behavior of the I-DIC routine, starting from an initial guess where each $d o f$ is initiated at $10 \%$ of the real value, identifying 8 EGP parameters, assuming $\mu$ and $\eta_{0,1}$ are known. (c) Converged relative error as a function of the relative initial guess, identifying 8 EGP material parameters. (d) Convergence behavior of the I-DIC routine, starting from an initial guess where each dof is initiated at $30 \%$ of the real value, identifying only the 4 rejuvenation EGP parameters, i.e. $S_{a}, r_{0}, r_{1}$ and $r_{2}$. After iteration 16, the 6 locked EGP parameters are released improving the identification of all parameters except $\eta_{0,1}$. The number of the particular iteration is depicted above the respective relative error values of subfigures (a) and (c), not converged procedures are indicated with $[-]$

reference solution. The 'distance' is specified as a relative error for each degree of freedom compared with the reference value.

Figure $7 \mathrm{~b}$ shows the convergence behavior for all 10 dof when initiated at $10 \%$ of the reference. It reveals that, during the first iterations where some dof tend to undergo large changes, relaxation is controlled by restraining the update $\delta\{\lambda\}$ to a maximum of $5 \%$ of the current value of the respective degree of freedom. After approximately 25 iterations the procedure has converged, resulting in the previously discussed accuracy. Figure 10a shows the final result for I-DIC procedures initialized at various distances from the reference solution. As expected, the final result does not depend on the initialization when it is close to the solution, however at an initial guess $\geq 20 \%$ error the procedure fails to converge.

From the analyses of the sensitivity fields and the DIC matrix, it was concluded that of the 10 EGP parameters, three parameters have reduced conditioning, namely $r_{1}, \mu$ and $\eta_{0,1}$. The proposed I-DIC method does not require that all constitutive parameters are included as dof. If the pressure-dependent parameter and the zero-viscosity are accurately known from other experiments, then these parameters can be locked to known values. Figure 10b shows the convergence history of the I-DIC procedure initiated at $10 \%$ of the reference solution, identifying the remaining 8 EGP parameters. By excluding the 2 dof, the convergence rate is noticeably improved. Additionally, the accuracy of the remaining 8 parameters is improved, as shown in Figure 10c. However, the convergence radius did not increase. Again, initial guesses $\geq 20 \%$ distance to the solution fail to converge. 


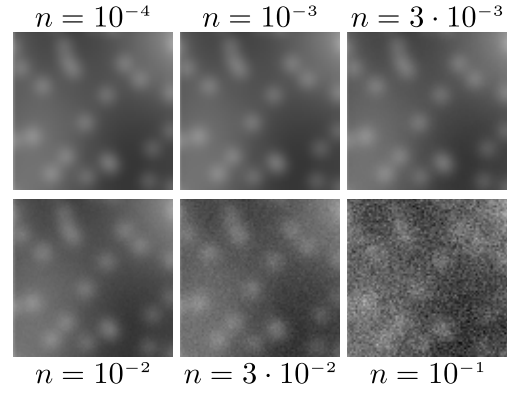

(a)

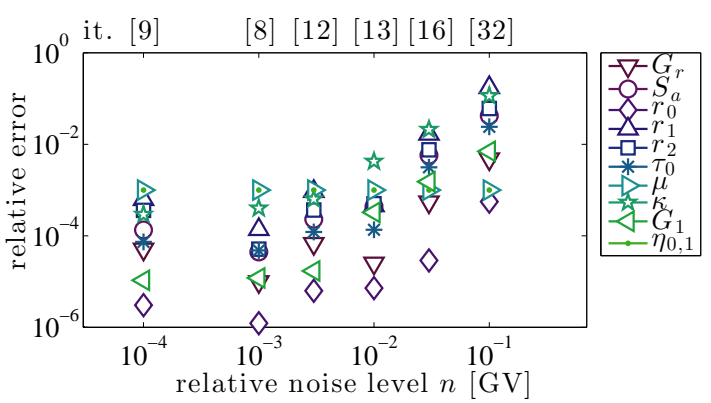

(b)

Figure 11. (a) Small sections of the pattern with various noise levels. (b) Sensitivity of the identification procedure as a function of the noise level $n$ (relative to the RMS of the pattern). Eight of the ten parameters are identified while $\mu$ and $\eta_{0,1}$ are assumed known.

Reducing the parameter space further, only the four aging/rejuvenation parameters can be chosen as the identification targets. A similar analysis is performed where the other six parameters are locked at $101 \%$ of their respective reference value (i.e. all including a small error). Now with only four dof the initial guess robustness improves significantly. Figure 10d shows the convergence history where these four parameters are initialized at a distance of $30 \%$ from the reference, while the remaining 6 parameters are frozen. In this state, with four dof, the procedure required 15 iterations to converge, after which the remaining 6 parameters are released continuing the procedure with $10 \mathrm{dof}$. Using such a two-step iterative scheme allowed the I-DIC procedure to converge from an imperfect initial guess and achieve an accuracy of within $1 \%$ of their expected value for most parameters.

\subsection{Noise sensitivity}

To investigate the sensitivity of the method to acquisition noise, artificial white noise is added to all images (i.e. the entire stack of images). For this particular test case 8-bit images are used, providing 256 integer gray values $(\mathrm{GV})$. The applied noise levels $n$ are given in terms of the standard deviation of the applied Gaussian noise field multiplied with the Root Mean Square (RMS) of the pattern (i.e. RMS = 122 [GV], see Figure 11a). Only 8 dof will be identified, assuming that $\mu$ and $\eta_{0,1}$ are known and set to their reference value. Figure $11 \mathrm{~b}$ shows the converged relative error for each parameter for various levels of image noise. For noise levels below $1 \%$ the solution is largely unaffected. The method remained robust up to a standard noise level of $10 \%$ even though the accuracy is affected.

\subsection{I-DIC for EGP identification}

A typical identification procedure for the EGP model requires many experiments, each using separate samples to identify parameters individually (e.g. one DMTA test plus 168 compression tests are used per polymer [47]). This is not only cumbersome, but identifying the historydependent aging parameter is troublesome, since it requires precise control of the sample variations over the required batch of samples. The proposed I-DIC method showed the ability to identify all 10 EGP parameters under the condition that a reasonable initial guess is provided. The convergence radius can be improved by distinguishing between parameters with high sensitivity and others that have less sensitivity. Accordingly, accuracy improves when the pressure-dependent parameter $\mu$ and the zero-viscosity $\eta_{0,1}$ are known. Likewise the initial guess robustness improves when only the four aging/rejuvenation parameters are targeted.

The applied experiment in this example was not optimized for EGP model parameter identification, and from the a priori analyses of the sensitivity fields it was already diagnosed that the experiment would be less sensitive to specific parameters. The proposed I-DIC method enables the analysis and improvement of the experiment prior to actually performing 
it. Consequently, it is possible to optimize the experiment for the identification of certain parameters by changing the sample geometry or modifying the proposed load path, see also [52]. For instance, loading the sample first in compression and then in tension may improve the sensitivity to $\mu$ and applying greater variations in the applied strain rate may improve the sensitivity for $\eta_{0,1}$.

The perturbation factor was set to $\epsilon=0.01$ for this example. Such a relatively large factor was necessary because the EGP model is not always stable (as are glassy polymers), which resulted in poor convergence behavior in the FEM simulation. This problem was more pronounced for the less sensitive parameters. Setting the perturbation factor to smaller levels yielded too noisy sensitivity fields, which resulted in poor stability of the I-DIC routine. It is not required to use the same perturbation factor for each degree of freedom, and optimizing the perturbation factors for each dof individually may alleviate these problems but this was not investigated in this work.

A point of caution regarding this example is the use of a single Maxwell mode for the EGP model. Van Breemen et al. [46] have shown that to accurately describe PC in complex loading situations up to 17 Maxwell modes are required with 2 parameters per mode. Identifying an EGP model with 42 parameters will definitely require optimization of the experiment for which the I-DIC method is to be used. Specifically the range of activated time scales should be expanded to encompass several decades of strain-rates.

\section{BULGE TESTS OF METAL-ELASTOMER MEMBRANES}

Instead of identifying multiple constitutive parameters of a single material, it is also possible to identify constitutive parameters of heterogeneous samples. For instance, the material response of micro-scale specimens where the geometrical length scale interacts with the intrinsic microstructural length scales tends to deviate from the bulk material response [53, 54] and is influenced by neighboring materials or phases $[55,56]$. When the material response depends on its environment or the manufacturing process, it is important to identify the materials closely to the situation where they are applied in the device. Examples of this are the structured metal interconnects adhered to elastomer substrates as encountered in stretchable electronic applications [57-59].

\subsection{Virtual bulge test experiment}

The presented test case consists of a $2 \mu \mathrm{m}$ thick elastomer membrane (surface area: $1 \times 6 \mathrm{~mm}^{2}$ ) with a line of high purity aluminum (Al) deposited on top of the elastomer membrane. The Al film has a thickness of $200 \mathrm{~nm}$, and is $100 \mu \mathrm{m}$ wide covering the full $6 \mathrm{~mm}$ length of the sample. The latter is loaded in a bulge test setup, where a pressure difference is applied causing the membrane to bulge outwards (see Figure 12a). The bulge profile (including the surface roughness) is measured using optical confocal microscopy.

A virtual experiment is used to perform an accurate assessment of the accuracy of the method, while excluding unknown experimental influences. Due to the slender aspect ratio of the membrane $(b \gg a)$, it is assumed that the strain in the $y$-direction (Figure 12a) is negligible. Therefore, the virtual experiment is modeled with $6432 \mathrm{D}$ plane strain quadrilateral elements which connect to 2653 nodes. For the elastomer substrate, thermoplastic urethane (TPU) is chosen, which is commonly found in stretchable electronic devices [59]. For the adopted bulge test, the strains in the substrate will remain below $10 \%$, therefore, TPU is modeled as a neo-Hookean medium with a single parameter, the modulus $C_{10}=3.3 \mathrm{MPa}$, see Figure 12b. Typically, the metal interconnects in these devices are produced using printed circuit board or lithographic techniques, often yielding high purity materials. Therefore, for this test case high purity aluminum, as discussed by Janssen et al. [60], is used and described by an elasto-plastic model with isotropic hardening, see Figure 12c. The FEM simulation is preformed in forcecontrol with 2000 steps to simulate 1 second of experimental time, of which 200 increments 


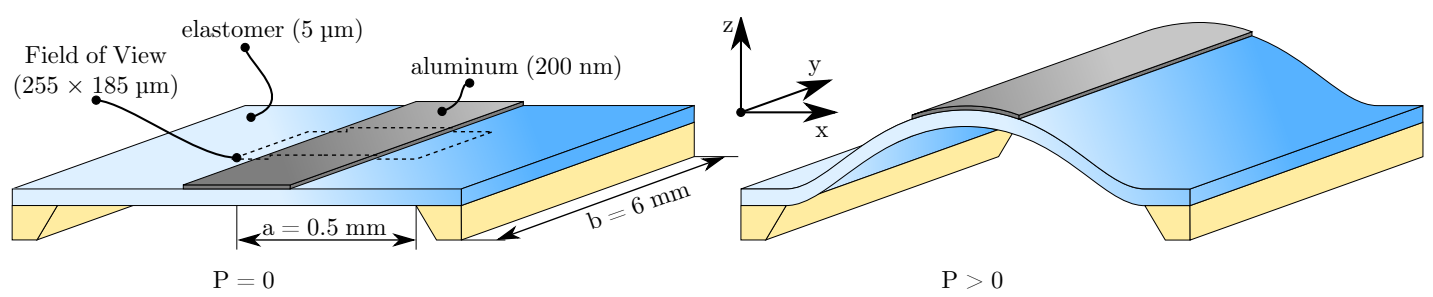

(a)

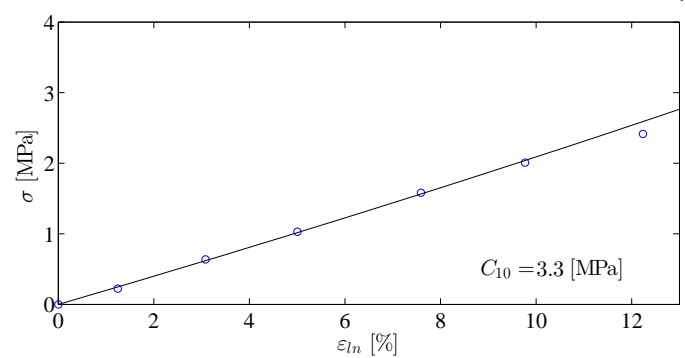

(b)

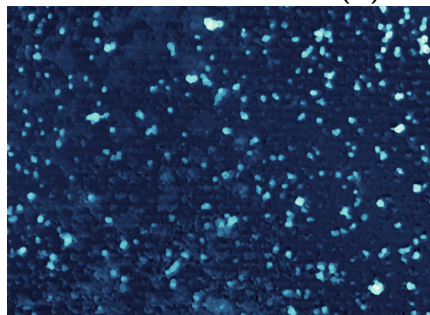

(d) $t=0$

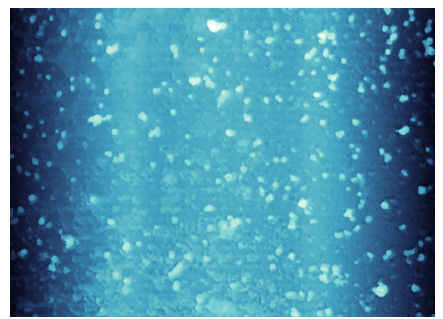

(e) $t=0.5 \mathrm{~s}$

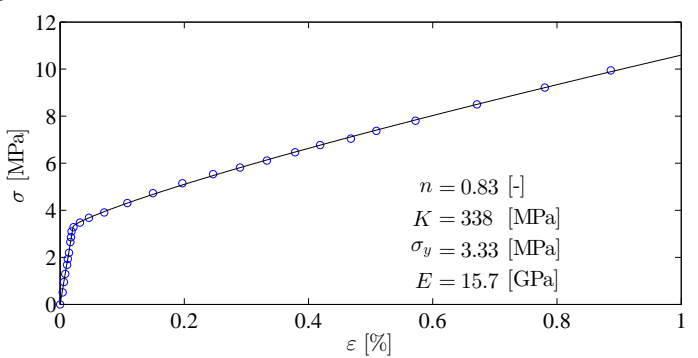

(c)

Figure 12. (a) Elastomer membrane with aluminum structure on top, deformed to a bulged shape by a pressure difference. (b) Experimental material response and the fitted models that will be used as a reference material in the virtual experiment for the TPU elastomer substrate described with a neoHookean model. (c) High purity aluminum following an elasto-plastic model with isotropic hardening.

$(d, e, f)$ Three image increments as applied in this example

at a regular $0.005 \mathrm{~s}$ interval are stored and used to generate the experimental images. See appendix A for details regarding the computational cost.

The applied pattern is an actual roughness of a bulge test measured with an optical confocal profilometer [61]. In this virtual experiment, the pattern and the out-of-plane displacement are measured by the profilometer. Consequently, the quasi-3D or topography correlation principle is applied in this example, i.e. $f$ now represents the height instead of the brightness. In this method the brightness/height conservation relation is relaxed by adding additional fields, which in this case describe the out-of-plane displacement

$$
f\left(\vec{x}, t_{0}\right)=f(\phi(\vec{x}, t,\{\lambda\}), t)+u_{z}(\vec{x}, t,\{\lambda\})+\eta(\vec{x}, t,\{\lambda\})
$$

where $u_{z}(\vec{x}, t,\{\lambda\})$ is the out-of-plane displacement field. Following the same procedure as in actual experiments [61], an extra set of basis functions is required, which within the proposed I-DIC procedure translate into an additional component in the sensitivity maps. Note that the displacement field and thus also each sensitivity map is a $3 \mathrm{D}$ vector field as a function of a $2 \mathrm{D}$ position vector, $\vec{x}$ and time, $t$. For instance, the displacement field is written as

$$
\vec{u}(\vec{x}, t,\{\lambda\})=u_{x}(\vec{x}, t,\{\lambda\}) \vec{e}_{x}+u_{y}(\vec{x}, t,\{\lambda\}) \vec{e}_{y}+u_{z}(\vec{x}, t,\{\lambda\}) \vec{e}_{z}, \quad \vec{x}=x \vec{e}_{x}+y \vec{e}_{y} .
$$

This additional field is naturally obtained by computing the derivative of the $z$-component of the displacement with respect to the degrees of freedom, using exactly the same formulation as expressed in Equation (9). 


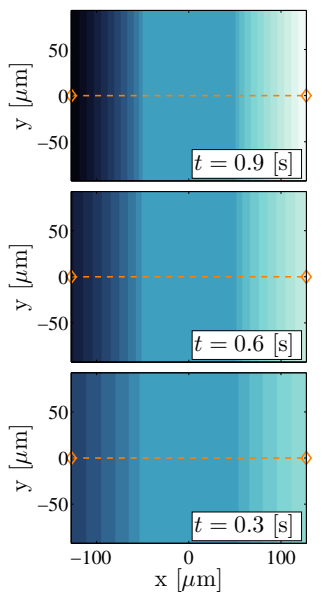

(a) $u_{x}$

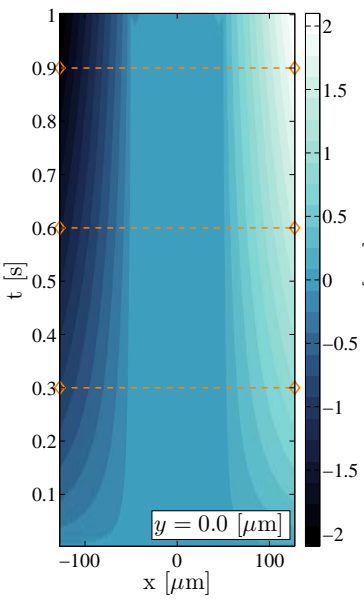

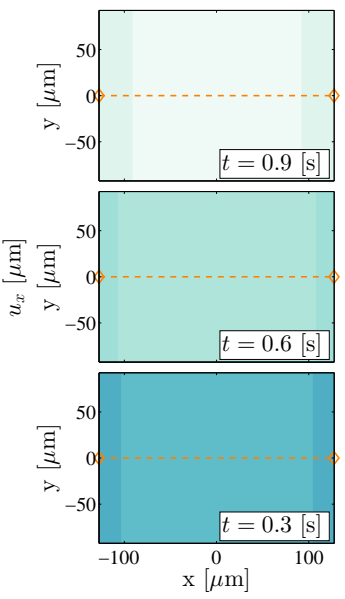

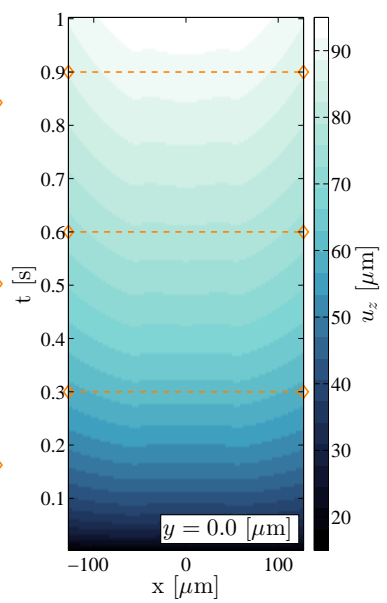

(b) $u_{z}$

Figure 13. (a) $x$-component and (b) $z$-component of the displacement field for three time increments and for a single $y$-plane and all time increments

The data obtained from confocal profilometry can be seen as digital images, where the gray values have the physical meaning of height. Additionally, the gray value discretization is not simply digitized in 8 bits (or similar). The height values are obtained through the interpretation of many confocal images, discretized as 32-bit floating point numbers. For this particular profilometer (Sensofar $\mathrm{pl} \mu 2300$ ) with a $20 \times$ objective applied in confocal mode, the images contain $557 \times 768$ pixels with a field of view of $255 \times 185 \mathrm{\mu m}^{2}$ (see Figure 12d-f).

\subsection{Parameter sensitivity}

Due to the plane strain nature of this experiment, there are no variations in the sensitivity fields along the $y$-direction, since $u_{y}(\vec{x}, t,\{\lambda\})=0$. Consequently, the data is presented in a $2 \mathrm{D}$ $x$-t-figure, taken at a single $y$-plane. For instance, the $x$ - and $z$-components of the displacement are shown in Figure 13ab.

For this example case, the experimental force is the pressure driving the bulge test. This pressure is included in the FE simulations as a load controlled boundary condition. Therefore, it is not required to add the force to the minimization procedure in the form of an extra potential (such as discussed in Section 2.2). As a result, the sensitivity fields only have displacement components in $x$ and $z$.

Figure 14 shows the scaled sensitivity fields applied in the I-DIC procedure. Both $x$ - and $z$-components of one sensitivity field are scaled with the same scaling constant (i.e. $\hat{\lambda}_{i}$ ) shown above the respective field. As before, the sensitivity fields can be used to diagnose potential identification problems. The difference in sensitivity is best expressed in the scaling constants $\hat{\lambda}_{i}$. The first observation is the contrast in sensitivity between the first degree of freedom $C_{10}$ and the other parameters. The observed contrast in sensitivity was expected since the elastomer substrate, which is much larger than the field of view, has a large impact on the displacement field. Moreover, the location of the aluminum strip, as well as the onset of plasticity can be recognized in the profile of the sensitivity fields. These maps reveal adequate sensitivity for all parameters, even though, the identification will be less sensitive to the Young's modulus $E$ and the hardening modulus $K$, with the lowest values $\hat{\lambda}_{2}=0.34$ and $\hat{\lambda}_{4}=0.80$. A spectral analysis similar to that discussed in the previous example (Section 3.3) can also be performed. However, for this case the sensitivity maps already reflect the correct sensitivity balance between the five degrees of freedom.

The proposed I-DIC method is applied to identify the five constitutive parameters, where the initial guess is taken equal to $130 \%$ of the reference values for the material parameters (listed 

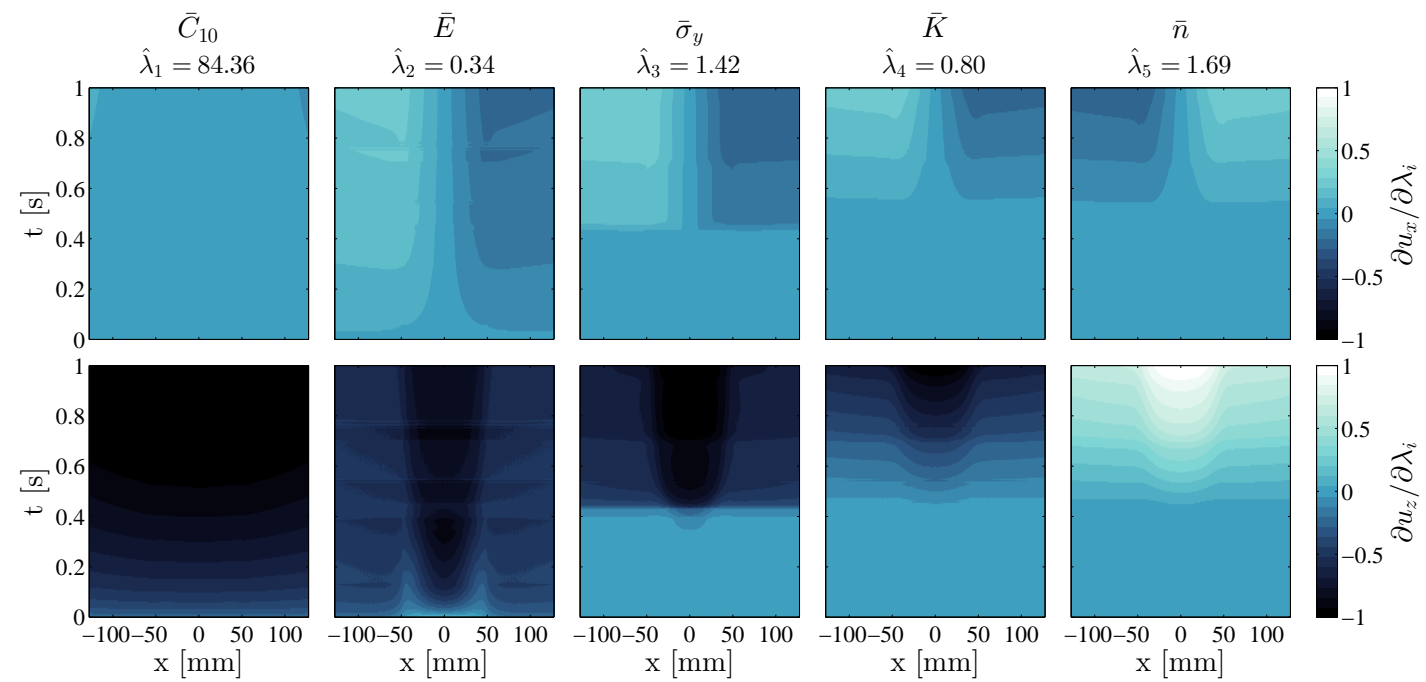

Figure 14. The scaled sensitivity maps (or basis functions) for all five unknown material parameters, for a single $y$-plane $(y=0.0 \mu \mathrm{m})$. Both figures in one dof column belong to one sensitivity field. The values of the scaling parameters $\hat{\lambda}_{i}$ are shown on top of the respective sensitivity field

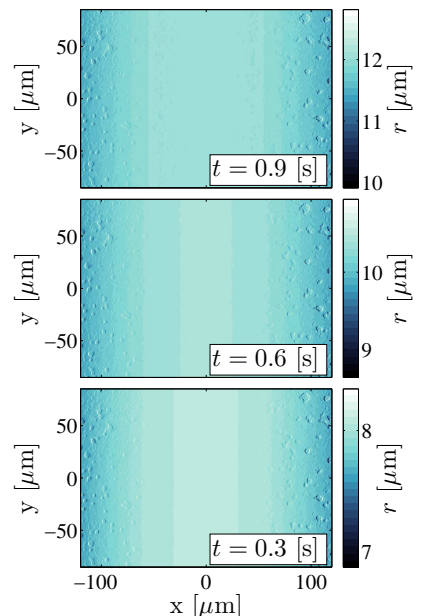

(a)

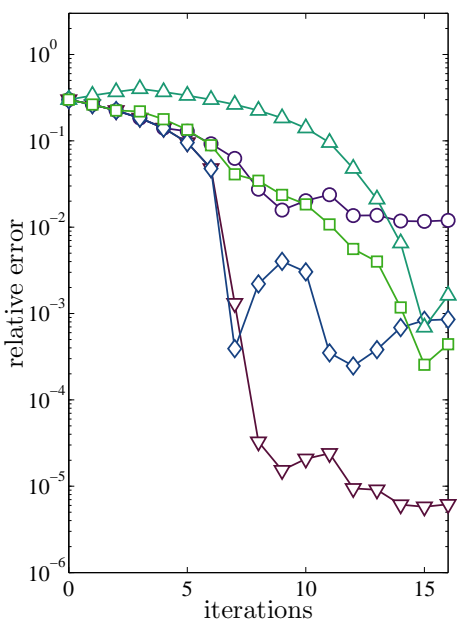

(b)
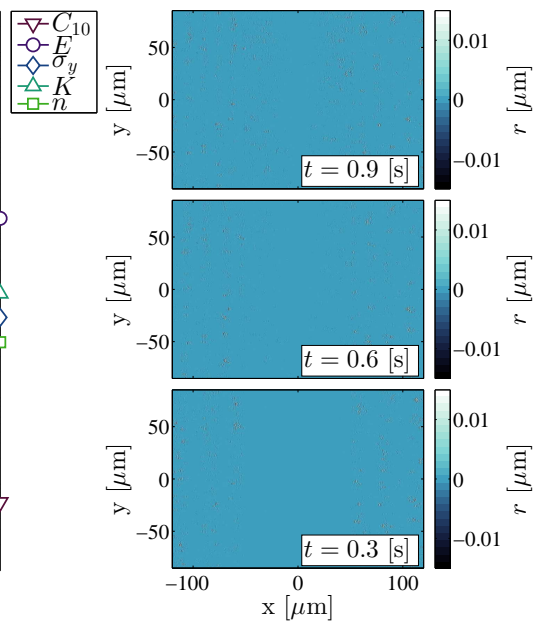

(c)

Figure 15. (a) Three initial residual images (i.e. based on the initial guess of $130 \%$ relative to the reference). (b) Convergence of the I-DIC method in terms of relative error for the bulge test. (c) Three corresponding final residual images. Note the difference in scale bar values between the initial and final residuals

in Figures 12bc). As with the previous (EGP) example, the residual field (stack) is analyzed for present patterns in the residuals. Figure 15 shows the initial and final residuals. The residual vanishes almost completely, which is expected since no additional acquisition noise was added. The only remaining visible features in the residual are the artifacts left behind by the sub-pixel interpolation.

For this virtual experiment, the reference displacement field is again known, enabling for a direct comparison between the identified and reference displacement fields. Figure 16 reveals that the largest error in the displacement field is found in the aluminum strip in the elastic part of the experiment $\left(\mathcal{E}_{u_{z}}=-0.4 \mathrm{~nm}\right)$, which compared to the pixel size $(332 \mathrm{~nm})$ translates into approximately $10^{-3}$ pixel accuracy. Compared with conventional DIC algorithms, where 


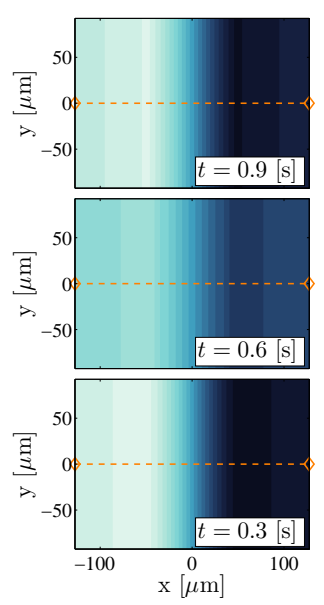

(a) $\mathcal{E}_{u_{x}}$

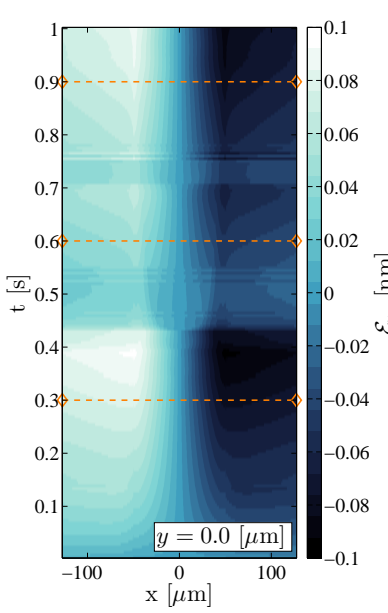

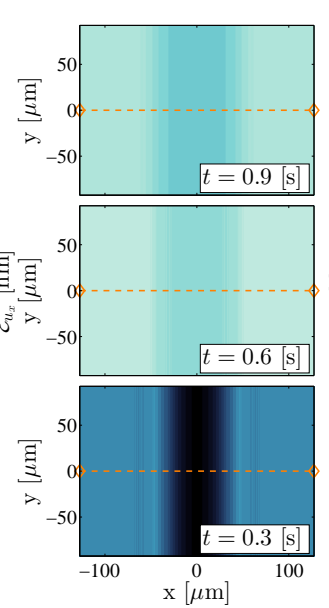

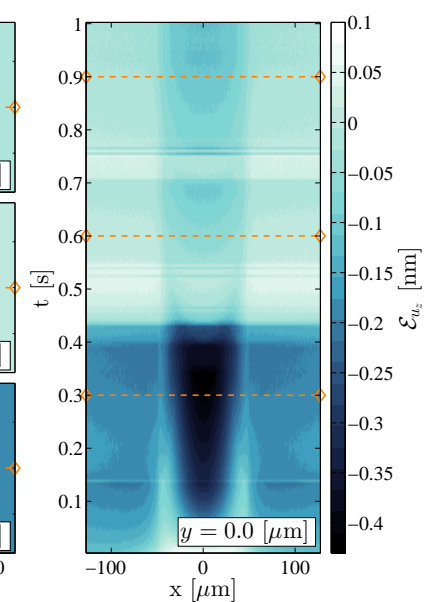

(b) $\mathcal{E}_{u_{z}}$

Figure 16. (a) $x$-component and (b) $y$-component of the displacement error field for three time increments, for a single $y$-plane and all time increments (in $\mathrm{nm}$ )

typically a displacement error of $10^{-2}$ pixel is considered as good [21], the achieved adequate accuracy of the proposed method is noteworthy (remembering that the accuracy would decrease if a model error were present).

Figure $15 \mathrm{~b}$ shows the iterative change of the relative error between the identified and the reference constitutive parameters. The procedure converges after 16 iterations, where the elastomer modulus and the yield stress $\left(C_{10}\right.$ and $\left.\sigma_{y}\right)$ converge first, followed by the other three parameters. This behavior is consistent with the analyses of the sensitivity maps. Additionally, the sensitivity maps predict that the Young's modulus and the hardening Modulus ( $E$ and $K$ ) are less sensitive, resulting in a reduced, yet still adequate, accuracy (compared to the high displacement accuracy), reflected by the relative error in the converged state. Akin to the EGP example, the achieved accuracy in the material parameters emphasizes the high requirements on the displacement field resolution to obtain sufficiently accurate constitutive parameters.

\subsection{Robustness with respect to the initial guess}

The initial guess robustness is next analyzed for this bulge test example. A number of IDIC identification routines are performed at various distances to the reference values for the constitutive parameters. Figure 17 shows the relative error for all dof in the converged state. As expected, the obtained values do not depend on the initial guess for small to moderate distances to the solution. The results show that this example case is more robust than the EGP case (i.e. a larger convergence radius), and converged for all tested initial guess distances, with the largest tested radius at $170 \%$. An improved robustness was expected since this example has less dof that are more strongly expressed in the sensitivity maps.

\subsection{Noise sensitivity}

The higher robustness of this example (relative to the EGP example) is also reflected in the noise sensitivity. Figure 17 shows the relative error for all dof in the converged state where additional noise is added to all images (i.e. in the entire stack). Different noise levels are added, again defined as the standard deviation of the white noise multiplied by the RMS value of the roughness $(1.5 \mu \mathrm{m})$ in the reference image. The accuracy of the identified parameters remains preserved for noise levels up to $3 \%$. Later on an increase in error is observed for increasing noise levels. The unexpected minimum at $10 \%$ is considered as accidental. Overall, the noise sensitivity for the example is remarkably low. This is due to the quasi-3D nature of the bulge test method and the use of profilometry data. The out-of-plane displacements 


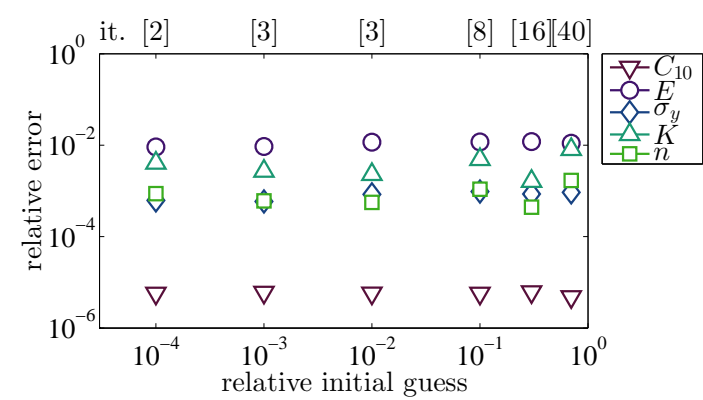

(a)

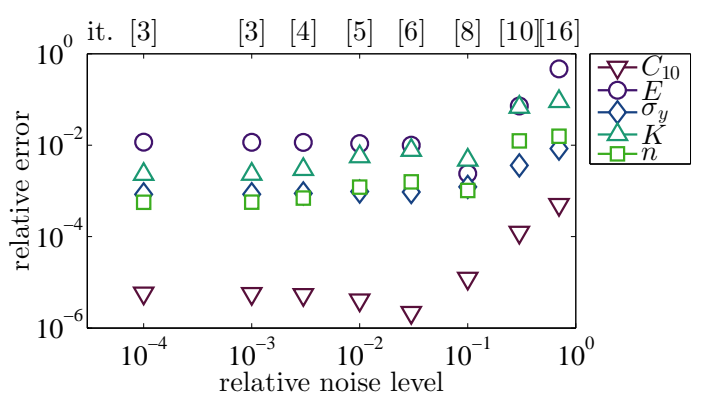

(b)

Figure 17. (a) Converged relative error of the I-DIC method applied to the bulge test case for various initial guesses. The relative initial guess is defined as the relative distance of all dof to the reference value. (b) Converged relative error for different levels of acquisition noise. The relative noise level is defined as standard deviation of the white noise scaled with the RMS of the DIC pattern. The number of iterations is shown above the respective relative error values

due to the bulging of the membrane enter the I-DIC procedure through a cylindrical shape in the roughness pattern, see Figure 12e-f. This cylinder is more pronounced than the sample roughness pattern, thereby stabilizing the iterative procedure. Close to the solution, the surface roughness pattern is required to provide sensitivity to the smaller levels of in-plane displacements.

\subsection{Model error}

Both discussed examples are cases where the same FE model is used in the virtual experiment and the forward simulation of the I-DIC routine. The result is that the model chosen for parameter identification can exactly describe the material of the experiment, i.e. there is no model error. The absence of model error allows the evaluation of the performance of the method only, excluding the additional uncertainty due to the model error. Indeed, any constitutive model is only an approximation of a real material, generally only a good approximation within a limited range of applicability. Consequently, a case without model error like those discussed so far will be highly unlikely when identifying a material model on real experimental data. Model errors deserve a careful examination but this is not addressed in this paper, to properly extract exclusively the role of the identification method. Nevertheless, an example is presented to highlight some tools to be used to detect and analyze such errors.

To induce some model error for this test case, the same virtual images are used, but the model to be identified for the aluminum is changed to a linear elastic one. The identification of the two elastic models is performed 8 times, each time including a larger part of the 200 images. Initially, the experiment behaves elastically, while at some point plasticity will set in. Consequently, the magnitude of the model error is expected to increase when including more images in the identification process.

Figure 18a shows three time-slices from the residual field as obtained by the identification procedure of the two elastic material models while using all 200 images. The figure shows a mostly positive residual for $t=0.3 \mathrm{~s}$ and a mostly negative residual for $t=0.9 \mathrm{~s}$. Additionally, an imprint of the pattern is clearly visible in both time-slices. More importantly, the range of the residual values is significant (i.e. $0.4 \mu \mathrm{m}$ ) when compared to the dynamic range of the pattern (i.e. $1.5 \mu \mathrm{m}$ ). These observations indicate that the image registration was compromised. For this particular case the residual field operates in the same direction as the out-of-plane displacement $u_{z}$. Accordingly, a positive residual indicates an overestimation of the out-ofplane displacement, namely, an underestimation of the sample stiffness, and vice versa.

The aluminum strip in the center of the sample is stretched by the substrate. Consequently, the strip will be loaded mostly at the left and right sides, which causes plasticity to occur first 

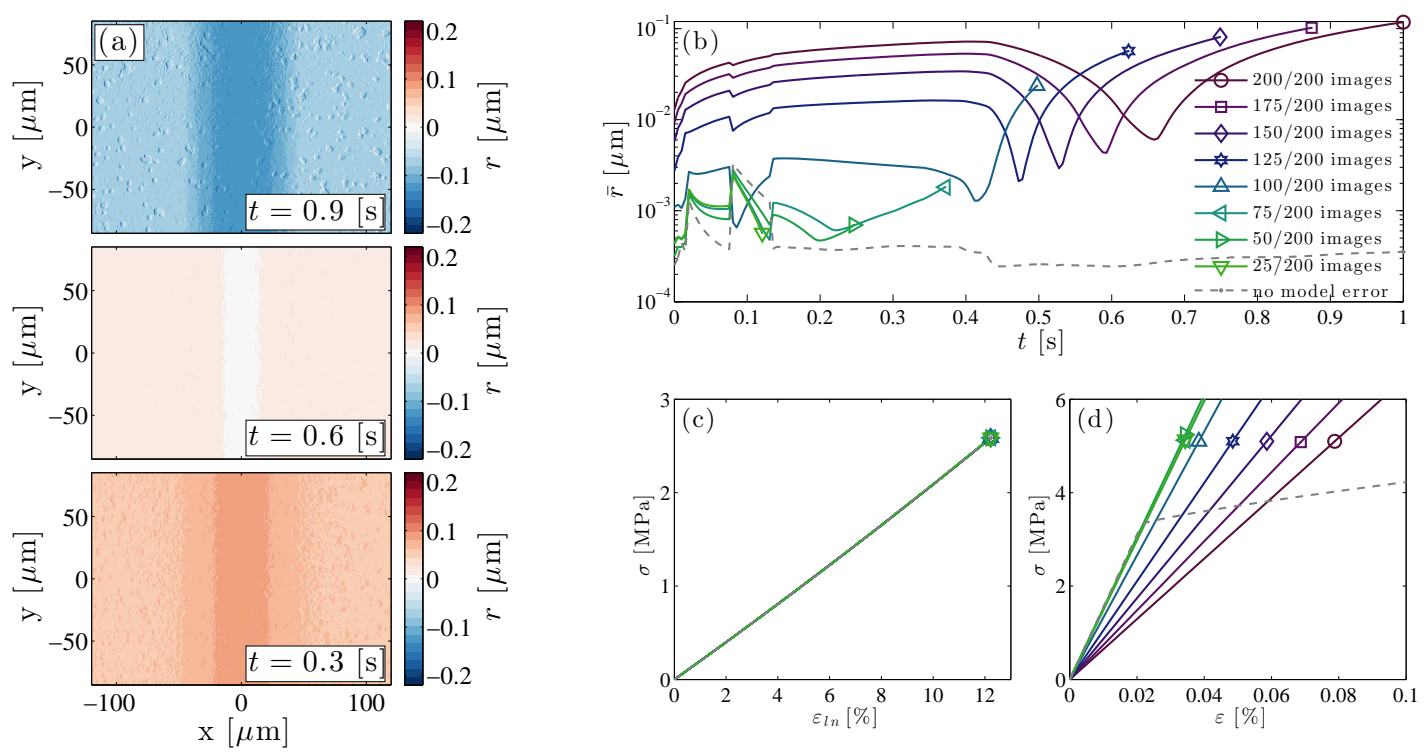

Figure 18. (a) Three image slices at three instances of time as a result of identifying two elastic models on an experiment that exhibited plasticity. (b) The mean absolute residual per image $\bar{r}$ as a function of time using various numbers of images for the identification. (c,d) The stress-strain curves expressed by the dof of the substrate, as identified using the same sets of images, as used for subfigure b. (c) and the aluminum coating $(\mathrm{d})$. The identification results without model error are included as a reference

Table II. Identified elastic parameters in the presence of model error when using an increasing amount of images

\begin{tabular}{lrccccccccc}
\hline number of images & $\mathbf{2 5}$ & $\mathbf{5 0}$ & $\mathbf{7 5}$ & $\mathbf{1 0 0}$ & $\mathbf{1 2 5}$ & $\mathbf{1 5 0}$ & $\mathbf{1 7 5}$ & $\mathbf{2 0 0}$ & no model error \\
\hline$C_{10}$ & {$[\mathbf{M P a}]$} & 3.3 & 3.3 & 3.3 & 3.3 & 3.3 & 3.299 & 3.298 & 3.296 & 3.3 \\
$E$ & {$[\mathbf{G P a}]$} & 15.4 & 15.3 & 14.9 & 13.3 & 10.5 & 8.7 & 7.4 & 6.5 & 15.5 \\
\hline
\end{tabular}

at the edges of the strip and gradually progresses inward. As a result, the influence of plasticity is introduced gradually and increases monotonically during the experiment. For the initial part of the experiment, the considered elastic material models should be a good approximation of the materials. Figure 18b, shows the residual averaged over a time-slice $\bar{r}$ as a function of time. The figure shows 9 curves, belonging to 8 identification procedures each using an increasing number of images, and the $9^{\text {th }}$ reveals the averaged residual for an identification without model error. The first three residual curves (i.e. up to 75 images) behave similarly, and remain within acceptable absolute values for the residual. Starting from the fourth curve (i.e. using 100 images or more) the trend changes and a dramatic up-sweep is observed for the latter part of the experiment (i.e. $t>0.4 \mathrm{~s}$ ). This is expected since the elastic models cannot describe the behavior observed in the later stage of the experiment. At approximately $3 / 4$ of the included experiment time a dip in the residual is apparent in figure 18b. Due to the Quasi-3D nature of this test case, any out-of-plane displacement which is not identified correctly will remain in the residual. The out-of-plane displacement of the elastic model intersects the experiment displacement at one point in time causing a minimum in the residual.

Figure 18c and 18d shows the stress strain curves of the two material models for the dof identified on the 8 image sequences as shown in Table II. Interestingly, as is apparent from Figure $18 \mathrm{c}$, the identification of the Mooney parameter $C_{10}$ is not significantly compromised by the model error. This is most likely because of the high sensitivity of this parameter compared to the other ones. Conversely, Figure 18d shows that only for identifications on the basis of the initial images (i.e. up to frame 75) the Young's modulus of aluminum is correctly retrieved, otherwise the modulus is underestimated. 


\subsection{Bulge test discussion}

The bulge test was chosen to prove that the proposed I-DIC method can be applied to identify the constitutive parameters of multiple materials in one sub-structured monolithic sample. This is important, especially for micro-electronics applications, where different materials shaped in thin and small structures deviate in properties from their bulk counterparts. The identification of these parameters is a challenge at that scale. The presented example shows that the adopted identification procedure is robust (i.e. mildly sensitive to the initial guess and acquisition noise). Additionally, this example showed the relative ease with which the proposed I-DIC method was extended to incorporate quasi-3D topographical profilometry data. The additional components required in the sensitivity maps naturally follow from the differentiation of the FE displacement fields with respect to the sought $d o f$.

For this test case the impact of model error is investigated by attempting to identify two elastic material models on an experiment where plasticity is present. This illustrates how a model error can manifest itself in an identification process. Therefore, it should be considered as an illustrative example rather than as a general result. However, some qualitative conclusions can be made. On the one hand, if the difference between the to-be-identified material model and the sample behavior is small, i.e. the model error is small, the impact on the displacement field will also be small and the identification will be mostly unaffected. On the other hand, when the displacement field simulated with the material model does not conform with the kinematics of the experiment, i.e. the model error is large, then the identification process will degrade. For these cases the residuals will increase, typically local in space and time. Far more work is needed to explore the various manifestations of model errors, their detection and circumventing strategies.

\section{FROM IMAGES TO MATERIAL PROPERTIES: CONCLUSIONS}

An Integrated Digital Image Correlation (I-DIC) method has been proposed, which relies on a bidirectional information transfer from images to the identification of the constitutive parameters, and reversely from the mechanics to the images by regularizing the DIC problem with "mechanically" admissible fields (implying kinematic and static admissibility as well as the obedience to a given constitutive law). These fields, sometimes called basis functions or shape functions, are not chosen manually, but obtained by computing the sensitivity of the displacement with respect to each sought degree of freedom. The proposed way to compute these sensitivity fields is by numerical differentiation of the displacement fields obtained from Finite Element (FE) simulations. This requires $n+1$ simulations, one for the current guess of the degrees of freedom and one perturbed simulation for each degree of freedom.

This unique way of applying FE-computed sensitivity fields as the DIC basis functions maximizes the regularization of the (ill-posed) DIC problem, thereby limiting the solution to the 'deformation modes' defined by the unknown material parameters. Moreover, the construction of the sensitivity fields is only limited by the types of problems that can be simulated in an FE setting. In particular, the second example (Section 4) showed that, extensions from 2D to quasi-3D (registration of height topographies) or to 3D is a small step, and merely requires FE simulations to be performed with adequate dimensions. Moreover, the tight integration with FEM has the additional benefit that the identified constitutive model is directly available for further application, for which it was originally developed, e.g. materials research, design optimization.

The accuracy of the method was discussed for two examples. The first example demonstrated that it is possible to identify a complex 10 parameter history- and rate-dependent glassy polymer model by only analyzing the captured images of a single, uniaxial tensile experiment. In spite of the simplicity of the experiment, the constitutive law gave rise to a propagating localized strain rate front. The second example demonstrated the possibility to identify the parameters of two materials in a monolithic sample tested in a (bulge) experiment. This 
is particularly useful for small scale testing where it is difficult to separate the individual materials, and where the material properties strongly depend on the manufacturing process and structural dimensions. However, it should be noted that those are just two examples and the boundaries of the application regime of the presented I-DIC method are yet to be explored.

The presented test cases were based on virtual experiments and did not include any model error. In real life, model errors cannot be avoided. It is difficult to imagine a convincing test case that would be representative of such model errors, and hence the consequences of identifying the parameters of an approximate model are not addressed herein. Yet, because this question is essential, it deserves some comments. The residual fields, which are part of the proposed I-DIC method, will be instrumental for the identification of model errors. If the residual fields contain areas, which may be local in space and time, where the level is higher than normally expected due to acquisition noise, then the registration has not been successful and may indicate significant model errors. Moreover, it is possible to compare the displacement field from the resulting FE simulation with those obtained from traditional full-field techniques, such as DIC, to assess if the identification was successful.

The inclusion of the data of all time increments into a single identification problem (from image to material property) is shown to be a powerful feature of the proposed method. It allows for the robust identification of history- or rate-dependent properties, and substantially enhances noise robustness. However, the data footprint increases exponentially, putting a high requirement on the available physical memory in the used computer system. Moreover, to create the sensitivity fields, one simulation has to be performed for each degree of freedom for each DIC iteration, which is computationally costly, yet easy to parallelize.

The finally obtained system is nonlinear, and possibly non-convex. For the minimization a Newton-Raphson algorithm is applied, which is known to converge rapidly when close to the solution, but easily captures a local (rather than a global) minimum. Therefore, the accuracy and efficiency of the method depend on the quality of the initial guess. This non-uniqueness problem was explored for the two example cases. Distances of $10 \%$ from the reference material parameters showed effortless convergence, while distances of $70 \%$ or more from the reference solution were still feasible. Other minimization techniques, such as the Levenberg-Marquardt method to name one, were not explored and may provide further improvements in terms of initial guess robustness.

It was demonstrated that, by analyzing the sensitivity fields, one can diagnose sensitivity problems before an experiment is actually performed. Similar to e.g. FEMU, VFM, and other full-field identification methods, the experiment no longer needs to trigger uniform strain or stress fields, it is possible to optimize the envisioned experiment by modeling it via $\mathrm{FE}$ simulations and analyzing the effect of certain design choices on the sensitivity fields. The latter ones do not yield insight into the influence of the pattern. Therefore, to diagnose possible sensitivity problems in relation to limitations of the DIC pattern, the DIC matrix can be analyzed, which is constructed from the sensitivity fields and the gradient of the pattern. An eigenvalue and eigenvector decomposition of the DIC matrix aptly identifies which degrees of freedom will be less sensitive. Finally, after convergence is reached, the residual fields have to be analyzed. If the proposed constitutive model does not fit the tested material (i.e. there is a significant model error), the dissident kinematics will limit the minimization of the residual field, which will leave systematic patterns behind in the residual field.

To conclude, the proposed method can be applied to a wide range of experiments, where many of the choices made in defining the experiment will impact the accuracy of the method. The proposed method gives an abundance of freedom, which is favorable for the applicability of the method, but which also puts a great deal of responsibility in the hands of the user. The sensitivity fields, DIC matrix and correlation residual fields enable for reasonable interpretations of the quality of the identification method for the particular case at hand, however they do not guarantee unconditional success, as for any ill-posed problem in Hadamard's sense. 


\section{ACKNOWLEDGEMENTS}

L.C.A. van Breemen is gratefully acknowledged for his support and input in this research, especially regarding the implementation and discussions regarding the EGP model. This work has been supported by the Dutch Technology Foundation (STW) and the Dutch Organization for Scientific Research (NWO).

\section{REFERENCES}

[1] Lemaitre J, Chaboche J. Mechanics of Solid Materials. Cambridge University Press: Cambridge (UK), 1990.

[2] Meuwissen MHH, Oomens CWJ, Baaijens FPT, Petterson R, Janssen JD. Determination of the elasto-plastic properties of aluminium using a mixed numericalexperimental method. Journal of Materials Processing Technology 1998; 75(1-3):204-211.

[3] Bonnet M, Constantinescu A. Inverse problems in elasticity. Inverse Problems 2005; 21(2):R1R50.

[4] van den Bosch MJ, Schreurs PJG, Geers MGD. Identification and characterization of delamination in polymer coated metal sheet. Journal of the Mechanics and Physics of Solids 2008; 56(11):3259-3276.

[5] Grédiac M, Hild F. Full-Field Measurements and Identification in Solid Mechanics. ISTE / Wiley: London (UK), 2012.

[6] Mottershead JE, Friswell MI. Model updating in structural dynamics: a survey. Journal of sound and vibration $1993 ; \mathbf{1 6 7}(2): 347-375$.

[7] Ahmadian H, Mottershead JE, Friswell MI. Regularisation methods for finite element model updating. Mechanical Systems and Signal Processing 1998; 12(1):47-64.

[8] Titurus B, Friswell MI. Regularization in model updating. International Journal for Numerical Methods in Engineering 2007; 75:440-478.

[9] Avril S, Bonnet M, Bretelle AS, Grédiac M, Hild F, Ienny P, Latourte F, Lemosse D, Pagano S, Pagnacco E, et al.. Overview of Identification Methods of Mechanical Parameters Based on Full-field Measurements. Experimental Mechanics 2008; 48(4):381-402.

[10] Mottershead JE, Link M, Friswell MI. The sensitivity method in finite element model updating: A tutorial. Mechanical Systems and Signal Processing 2011; 25(7):2275-2296.

[11] Wang W, Mottershead JE, Ihle A, Siebert T, Reinhard Schubach H. Finite element model updating from full-field vibration measurement using digital image correlation. Journal of Sound and Vibration 2011; 330(8):1599-1620.

[12] Wang W, Mottershead JE, Sebastian CM, Patterson EA. Shape features and finite element model updating from full-field strain data. International Journal of Solids and Structures 2011; 48(1112):1644-1657.

[13] Chu TC, Ranson WF, Sutton MA. Applications of digital-image-correlation techniques to experimental mechanics. Experimental mechanics 1985; 25(3):232-244.

[14] Sutton MA, Mingqi C, Peters III WH, Chao YJ, McNeill SR. Application of an optimized digital correlation method to planar deformation analysis. Image and Vision Computing 1986; 4(3):143150 .

[15] Bruck HA, McNeill SR, Sutton MA, Peters III WH. Digital image correlation using NewtonRaphson method of partial differential correction. Experimental Mechanics 1989; :261-267.

[16] Sutton MA, Turner JL, Bruck HA, Chae TA. Full-field Representation of Discretely Sampled Surface Deformation for Displacement and Strain Analysis. Experimental Mechanics 1991; 31(2):168-177.

[17] Pan B, Qian K, Xie H, Asundi A. Two-dimensional digital image correlation for in-plane displacement and strain measurement: a review. Measurement Science and Technology 2009; 20(6):062 001 . 
[18] Pan B, Asundi A, Xie H, Gao J. Digital image correlation using iterative least squares and pointwise least squares for displacement field and strain field measurements. Optics and Lasers in Engineering 2009; 47(7-8):865-874.

[19] Cheng P, Sutton MA, Schreier HW, McNeill SR. Full-field speckle pattern image correlation with B-spline deformation function. Experimental Mechanics 2002; 42(September).

[20] Schreier HW, Sutton MA. Systematic Errors in Digital Image Correlation Due to Undermatched Subset Shape Functions. Experimental Mechanics 2002; 42(3):303-310.

[21] Hild F, Roux S. Comparison of Local and Global Approaches to Digital Image Correlation. Experimental Mechanics 2012; 52(9):1503-1519.

[22] Réthoré J, Roux S, Hild F. An extended and integrated digital image correlation technique applied to the analysis fractured samples. Eur. J. Comput. Mech. 2009; 18:285-306.

[23] Kavanagh KT, Clough RW. Finite element applications in the characterization of elastic solids. International Journal of Solids and Structures 1971; 7:11-23.

[24] Gras R, Leclerc H, Roux S, Otin S, Schneider J, Périé JN. Identification of the Out-of-Plane Shear Modulus of a 3D Woven Composite. Experimental Mechanics 2012; 53(5):719-730.

[25] Grédiac M, Pierron F, Surrel Y. Novel Procedure for Complete In-plane Composite Characterization Using a Single T-shaped Specimen. Experimental Mechanics 1999; 39(2):142149 .

[26] Grédiac M, Pierron F. Applying the Virtual Fields Method to the identification of elasto-plastic constitutive parameters. International Journal of Plasticity 2006; 22(4):602-627.

[27] Pierron F, Vert G, Burguete R, Avril S, Rotinat R, Wisnom MR. Identification of the Orthotropic Elastic Stiffnesses of Composites with the Virtual Fields Method: Sensitivity Study and Experimental Validation. Strain 2007; 43(3):250-259.

[28] Pierron F, Grédiac M. The Virtual Fields Method. Springer New York: New York, NY, 2012.

[29] Leclerc H, Périé JN, Roux S, Hild F. Integrated digital image correlation for the identification of mechanical properties. Computer Vision/Computer Graphics CollaborationTechniques. Springer: Berlin Heidelberg, 2009; 161-171.

[30] Hild F, Roux S. Digital Image Correlation: from Displacement Measurement to Identification of Elastic Properties - a Review. Strain 2006; 42(2):69-80.

[31] Mathieu F, Hild F, Roux S. Image-based identification procedure of a crack propagation law. Engineering Fracture Mechanics 2013; 103:48-59.

[32] Baldi A. Residual Stress Measurement Using Hole Drilling and Integrated Digital Image Correlation Techniques. Experimental Mechanics 2013; 54(3):379-391.

[33] Blaysat B, Hoefnagels J, Lubineau G, Alfano M, Geers M. Interface debonding characterization by image correlation integrated with Double Cantilever Beam kinematics. International Journal of Solids and Structures 2014; doi:10.1016/j.ijsolstr.2014.06.012.

[34] Réthoré J. A fully integrated noise robust strategy for the identification of constitutive laws from digital images. International Journal for Numerical Methods in Engineering 2010; 84:631-660.

[35] Réthoré J, Muhibullah, Elguedj T, Coret M, Chaudet P, Combescure A. Robust identification of elasto-plastic constitutive law parameters from digital images using 3D kinematics. International Journal of Solids and Structures 2013; 50(1):73-85.

[36] Mathieu F, Leclerc H, Hild F, Roux S. Estimation of Elastoplastic Parameters via Weighted FEMU and Integrated-DIC. Experimental Mechanics 2014; doi:10.1007/s11340-014-9888-9.

[37] Lucas B, Kanade T. An iterative image registration technique with an application to stereo vision. 7th International Joint Conference on Artificial Intelligence, 1981; 674-679.

[38] Besnard G, Guérard S, Roux S, Hild F. A spacetime approach in digital image correlation: MovieDIC. Optics and Lasers in Engineering 2011; 49(1):71-81. 
[39] Schreier HW, Braasch JR, Sutton MA. Systematic errors in digital image correlation caused by intensity interpolation. Optical Engineering 2000; 39(11):2915-2921.

[40] Bay BK, Smith TS, Fyhrie DP, Saad M. Digital volume correlation: Three-dimensional strain mapping using X-ray tomography. Experimental Mechanics 1999; 39(3):217-226.

[41] Bay BK. Methods and applications of digital volume correlation. The Journal of Strain Analysis for Engineering Design 2008; 43(8):745-760.

[42] Fedele R. Simultaneous Assessment of Mechanical Properties and Boundary Conditions Based on Digital Image Correlation. Experimental Mechanics 2014; doi:10.1007/s11340-014-9931-x.

[43] Dufour JE, Hild F, Roux S. Integrated digital image correlation for the evaluation and correction of optical distortions. Optics and Lasers in Engineering 2014; 56:121-133.

[44] Tervoort TA, Klompen ETJ, Govaert LE. A multi-mode approach to finite, three-dimensional, nonlinear viscoelastic behavior of polymer glasses. Journal of Rheology 1996; 40(5):779-797.

[45] Meijer HEH, Govaert LE. Multi-Scale Analysis of Mechanical Properties of Amorphous Polymer Systems. Macromolecular Chemistry and Physics 2003; 204(2):274-288.

[46] van Breemen LCA, Klompen ETJ, Govaert LE, Meijer HEH. Extending the EGP constitutive model for polymer glasses to multiple relaxation times. Journal of the Mechanics and Physics of Solids 2011; 59(10):2191-2207.

[47] van Breemen LCA, Engels TAP, Klompen ETJ, Senden DJA, Govaert LE. Rate- and temperature-dependent strain softening in solid polymers. Journal of Polymer Science Part B: Polymer Physics 2012; 50(24):1757-1771.

[48] van Breemen LCA, Govaert LE, Meijer HEH. Scratching polycarbonate: A quantitative model. Wear 2012; 274-275:238-247.

[49] Klompen ETJ, Govaert LE. Nonlinear viscoelastic behaviour of thermorheologically complex materials. Mechanics of Time-Dependent Materials 1999; 3:49-69.

[50] Visser HA, Bor TC, Wolters M, Warnet LL, Govaert LE. Influence of physical aging on impact embrittlement of uPVC pipes. Plastics, Rubber and Composites 2011; 40(5):201-212.

[51] Engels TAP, van Breemen LCA, Govaert LE, Meijer HEH. Criteria to predict the embrittlement of polycarbonate. Polymer 2011; 52(8):1811-1819.

[52] Rossi M, Pierron F. On the use of simulated experiments in designing tests for material characterization from full-field measurements. International Journal of Solids and Structures $2012 ;$ 49(3-4):420-435.

[53] Nix WD. Mechanical properties of thin films. Metallurgical transactions A 1989; 20A:2217-2245.

[54] Vinci RP, Vlassak JJ. Mechanical behavior of thin films. Annual Review of Materials Science 1996; 26:431-462.

[55] Arzt E. Size effects in materials due to microstructural and dimensional constraints: a comparative review. Acta Materialia 1998; 46(16):5611-5626.

[56] Gruber PA, Böhm J, Onuseit F, Wanner A, Spolenak R, Arzt E. Size effects on yield strength and strain hardening for ultra-thin $\mathrm{Cu}$ films with and without passivation: A study by synchrotron and bulge test techniques. Acta Materialia 2008; 56(10):2318-2335.

[57] Rogers JA, Someya T, Huang YY. Materials and mechanics for stretchable electronics. Science (New York, N.Y.) 2010; 327(5973):1603-1607.

[58] Vanfleteren J, Loeher T, Gonzalez M, Bossuyt F, Vervust T, de Wolf I, Jablonski M. SCB and SMI : two stretchable circuit technologies, based on standard printed circuit board processes. Circuit World 2012; 38(4):232-242.

[59] Loeher T, Manessis D, Heinrich R, Vanfleteren J, DeBaets J, Ostmann A, Reichl H. Stretchable electronic systems. Electronics Packaging Technology Conference 2006; :271-276.

[60] Janssen PJM, Hoefnagels JPM, de Keijser TH, Geers MGD. Processing induced size effects in plastic yielding upon miniaturisation. Journal of the Mechanics and Physics of Solids 2008; $\mathbf{5 6}(8): 2687-2706$. 
[61] Neggers J, Hoefnagels JPM, Hild F, Roux S, Geers MGD. Direct stress-strain measurements from bulged membranes using topography image correlation. Experimental Mechanics 2014; 54(5):717727 .

[62] Rall LB. Automatic Differentiation: Techniques and Applications, Lecture Notes in Computer Science, vol. 120. Springer: Berlin, 1981.

\section{A. COMPUTATIONAL COST OF THE METHOD}

The computational cost for identifying the parameters using the proposed I-DIC method far outweigh the computational cost required for typical DIC implementations. However, the result of the I-DIC method is more comparable to a combined DIC and inverse identification method (e.g. FEMU), since it gives the displacement field and performs the material identification. It is expected that the computational costs of the I-DIC approach are comparable with those of a FEMU approach. For both presented examples most of the computational cost was spent on solving the forward FE simulations required to obtain the sensitivity fields, i.e. Equation (9).

For the polycarbonate example of Section 3, evaluating Equation (9) required 11 FE simulations, each lasting approximately 21 minutes, using 33 cores (3 per simulation using domain decomposition) on $11 \operatorname{Intel}(\mathrm{R}) \mathrm{Xeon}(\mathrm{R}) \mathrm{X} 5550$ quad-core processors. The remainder of the computational work of the iteration consumed another 9 minutes using 8 cores on 2 AMD Opteron(tm) 8431 hexa-core processors requiring approximately $9 \mathrm{~Gb}$ of memory. Consequently, the longest identification evaluated for this test case required almost 17 hours to converge (i.e. 31 iterations, see Figure $7 \mathrm{~b}$ ).

For the bulge test example of Section 4, evaluating Equation (9) required 6 FE simulations, each lasting approximately 9 minutes, using 12 cores (2 per simulation using domain decomposition) on 6 Intel(R) Xeon(R) X5550 quad-core processors. The remainder of the computational work of the iteration consumed another 3 minutes using 12 cores on 2 AMD Opteron(tm) 8431 hexa-core processors requiring approximately $40 \mathrm{~Gb}$ of memory. Consequently, the longest identification evaluated for this test case required 8 hours to converge (i.e. 40 iterations, see Figure 17a).

It may be fair to say that the computational cost was not yet considered to be a limitation, and hence no effort was made in terms of optimization. In contrast, the ultimate accuracy was the main objective. It is clear that a significant reduction of computation time can be achieved with a modest effort. For instance, an FE code that is compatible with Algorithmic Differentiation [62] would result in considerable savings.

\section{B. BRIEF DESCRIPTION OF THE EGP MODEL}

In the Eindhoven Glassy Polymer (EGP) model [45], the polymer behavior is modeled by two contributions acting in parallel. The first, $\boldsymbol{\sigma}_{s}$, is the viscoelastic contribution related to the intermolecular interaction that is responsible for the low-strain behavior including yield and strain softening. The second, $\boldsymbol{\sigma}_{r}$, is the contribution of the molecular network, responsible for strain hardening in the large-strain regime

$$
\boldsymbol{\sigma}=\boldsymbol{\sigma}_{s}+\boldsymbol{\sigma}_{r}
$$

The hardening stress $\boldsymbol{\sigma}_{r}$ is modeled as neo-Hookean [44]

$$
\boldsymbol{\sigma}_{r}=G_{r} \tilde{\boldsymbol{B}}^{d}
$$

with $G_{r}$ the strain hardening modulus, and $\tilde{\boldsymbol{B}}^{d}$ the deviatoric part of the isochoric left Cauchy-Green strain tensor. The inter-molecular stress is split into hydrostatic and deviatoric parts, the latter is typically modeled with a combination of $n$ parallel Maxwell elements

$$
\boldsymbol{\sigma}_{s}=\boldsymbol{\sigma}_{s}^{h}+\sum_{i=1}^{n} \boldsymbol{\sigma}_{s, i}^{d}=\kappa(J-1) \boldsymbol{I}+\sum_{i=1}^{n} G_{i} \tilde{\boldsymbol{B}}_{e, i}^{d}
$$

where, $\kappa$ is the bulk modulus, $J$ the determinant of the deformation gradient tensor (expressing volume changes), $\boldsymbol{I}$ the unity tensor, $G$ the shear modulus and $\tilde{\boldsymbol{B}}_{e}$ the elastic part of the isochoric left CauchyGreen strain tensor. For the example discussed in Section 3, the number of Maxwell elements equals 
one, making the summation unnecessary. The time and history dependence of the model is updated by introducing the time evolution of $\tilde{\boldsymbol{B}}_{e}$ and $J$

$$
\begin{aligned}
\dot{J} & =J \operatorname{tr}(\boldsymbol{D}), \\
\dot{\tilde{\boldsymbol{B}}}_{e, i} & =\left(\tilde{\boldsymbol{L}}-\boldsymbol{D}_{p, i}\right) \cdot \tilde{\boldsymbol{B}}_{e, i}+\tilde{\boldsymbol{B}}_{e, i} \cdot\left(\tilde{\boldsymbol{L}}^{c}-\boldsymbol{D}_{p, i}\right),
\end{aligned}
$$

where $\tilde{\boldsymbol{L}}$ is isochoric velocity gradient tensor and $\boldsymbol{D}_{p}$ the plastic deformation rate tensor. The latter is modeled with non-Newtonian flow rule with modified Eyring viscosity $\eta_{i}$

$$
\boldsymbol{D}_{p, i}=\frac{\boldsymbol{\sigma}_{s, i}^{d}}{2 \eta_{i}(T, \overline{\boldsymbol{\tau}}, p, S)} .
$$

The modified Eyring viscosity depends on the temperature $T$, the equivalent stress $\bar{\tau}$, the pressure $p$ and the strain softening $S$

$$
\eta_{i}(T, \overline{\boldsymbol{\tau}}, p, S)=\eta_{0, i} \frac{\overline{\boldsymbol{\tau}} / \tau_{0}}{\sinh \left(\boldsymbol{\tau} / \tau_{0}\right)} \exp \left[\frac{\mu p}{\tau_{0}}\right] \exp \left[S\left(\bar{\gamma}_{p}\right)\right]
$$

where the important parameters are $\eta_{0, i}$, the zero-viscosity; $\tau_{0}$, the characteristic stress; $\mu$, the pressure-dependent parameter. Finally, the model is completed through the softening function [46]

$$
S\left(\bar{\gamma}_{p}\right)=S_{a} \frac{\left(1+\left[r_{0} \exp \left(\bar{\gamma}_{p}\right)\right]^{r_{1}}\right)^{\left(r_{2}-1\right) / r_{1}}}{\left(1+r_{0}^{r_{1}}\right)^{\left(r_{2}-1\right) / r_{1}}}
$$

where $S_{a}$ captures the initial thermodynamic state that increases the yield stress with respect to the rejuvenated state of the material. The parameters $r_{0}, r_{1}$ and $r_{2}$ are three fitting parameters that control the shape of the softening response of the model as a function of the plastic strain $\bar{\gamma}_{p}$. 